\title{
Predicting the Neutron and Proton Masses Based on Baryons which Are Yang-Mills Magnetic Monopoles and Koide Mass Triplets
}

\author{
Jay R. Yablon \\ Schenectady, New York, USA \\ Email: jyablon@nycap.rr.com
}

Received February 14, 2013; revised April 19, 2013; accepted April 26, 2013

Copyright (C) 2013 Jay R. Yablon. This is an open access article distributed under the Creative Commons Attribution License, which permits unrestricted use, distribution, and reproduction in any medium, provided the original work is properly cited.

\begin{abstract}
We show how the Koide relationships and associated triplet mass matrices can be generalized to derive the observed sum of the free neutron and proton rest masses in terms of the up and down current quark masses and the Fermi vev to six parts in 10,000. This sum can then be solved for the separate neutron and proton masses using the neutron minus proton mass difference derived by the author in a recent, separate paper. The oppositely-signed charges of the up and down quarks are responsible for the appearance of a complex phase $\exp (\mathrm{i} \delta)$ and real rotation angle $\theta$ which leads on an independent basis to mass and mixing matrices similar to that of Cabibbo, Kobayashi and Maskawa (CKM). These can then be used to specify the neutron and proton mass relationships to unlimited accuracy using $\theta$ as a nucleon fitting angle deduced from empirical data. This fitting angle is then shown to be related to an invariant of the CKM mixing angles within experimental errors. Also developed is a master mass and mixing matrix which may help to interconnect all baryon and quark masses and mixing angles. The Koide generalizations developed here enable these neutron and proton mass relationships to be given a Lagrangian formulation based on neutron and proton field strength tensors that contain vacuum-amplified and current quark wavefunctions and masses. In the course of development, we also uncover new Koide relationships for the neutrinos, the up quarks, and the down quarks.
\end{abstract}

Keywords: Proton Mass; Neutron Mass; Baryons; Magnetic Monopoles; Koide; CKM Mixing Angles; Current Quarks; Constituent Quarks

\section{Introduction}

In an earlier paper [1] the author introduced the thesis that baryons are Yang-Mills magnetic monopoles. Using the t'Hooft magnetic monopole Lagrangian in (2.1) of [2] and a Gaussian ansatz for fermion wavefunctions from (14) of O'Hanian's [3] to obtain energies according to

$$
E=-\iiint \mathfrak{L}_{\text {gauge }} \mathrm{d}^{3} x=\frac{1}{2} \operatorname{Tr} \iiint F_{\mu v} F^{\mu v} \mathrm{~d}^{3} x,
$$

it became possible in Equation (11.22) of [1] to predict the electron rest mass as a function of the up and down quark masses, specifically:

$$
m_{e}=3\left(m_{d}-m_{u}\right) /(2 \pi)^{\frac{3}{2}},
$$

with the factor $(2 \pi)^{\frac{3}{2}}$ emerging from three-dimensional Gaussian integration. Based on a "resonant cavity" analysis of the nucleons whereby the energies released or retained during nuclear binding are directly dependent upon the masses of the quarks contained within the nucleons, it was also predicted that latent, intrinsic binding energies of a neutron and proton, see (12.12) and (12.13) of [1], are given by:

$$
\begin{aligned}
B_{P} & =2 m_{u}+m_{d}-\left(m_{d}+4 \sqrt{m_{u} m_{d}}+4 m_{u}\right) /(2 \pi)^{\frac{3}{2}} \\
& =7.640679 \mathrm{MeV}, \\
B_{N} & =2 m_{d}+m_{u}-\left(m_{u}+4 \sqrt{m_{u} m_{d}}+4 m_{d}\right) /(2 \pi)^{\frac{3}{2}} \\
& =9.812358 \mathrm{MeV} .
\end{aligned}
$$

These predict a latent binding energy of 8.7625185 $\mathrm{MeV}$ per nucleon for a nucleus with an equal number of protons and neutrons, which is remarkably close to what is observed for all but the very lightest nuclides, as well as a total latent binding energy of $493.028394 \mathrm{MeV}$ for ${ }^{56} \mathrm{Fe}$, in contrast to the empirical binding energy of 
492.253892 MeV. This is understood to mean that $99.8429093 \%$ of the available binding energy in ${ }^{56} \mathrm{Fe}$ is applied to inter-nucleon binding, with the balance of $0.1570907 \%$ retained for the intra-nucleon quark confinement. It was also noted that this percentage of energy released for inter-nucleon binding is higher in ${ }^{56} \mathrm{Fe}$ than in any other nuclide, which further explains that although the quarks come closer to de-confinement in ${ }^{56} \mathrm{Fe}$ than in any other nuclide (which also explains the "first EMC effect" [4]), they do always remain confined, as emphasized by the decline in this percentage for elements with nuclear weights higher than ${ }^{56} \mathrm{Fe}$.

In a second paper [5], the author showed how the thesis that baryons are Yang-Mills magnetic monopoles together with the foregoing "resonant cavity" analysis can be used to predict the binding energies of the $1 \mathrm{~s}$ nuclides, namely ${ }^{2} \mathrm{H},{ }^{3} \mathrm{H},{ }^{3} \mathrm{He}$ and ${ }^{4} \mathrm{He}$ to parts per hundred thousand for ${ }^{3} \mathrm{He}$ and in all other cases to parts per million, and also to predict the difference between the neutron and proton masses according to:

$$
M_{N}-M_{P}=m_{u}-\left(3 m_{d}+2 \sqrt{m_{\mu} m_{d}}-3 m_{u}\right) /(2 \pi)^{\frac{3}{2}} \text {. }
$$

This relationship, originally predicted in (7.2) of [5] to about seven parts per ten million in AMU, was later taken in (10.1) of [5] to be an exact relationship, and all of the other prior mass relationships which had been developed were then nominally adjusted at the seventh decimal place to implement (1.4) as an exact relationship. The review of the solar fusion cycle in Section 9 of [5] served to emphasize how effectively this resonant cavity analysis can be used to accurately predict empirical binding energies, and suggested how applying gamma radiation with the right resonant harmonics to a store of hydrogen may well have a catalyzing effect for nuclear fusion. This relationship (1.4) will also play an important role in the development here.

At the heart of these numeric calculations which accord so well with empirical data were the two outer products (4.9) and (4.10) in [5] for the neutron and the proton, with components given by (4.11) and related relationships developed throughout Sections 3 and 4 of [5]. In particular, the two matrices which stood at the center of these successful binding energy calculations were $3 \times$ 3 Yang-Mills diagonalized matrices $K$ of mass dimension $\frac{1}{2}$ with components $\operatorname{diag}\left(K_{N}\right)=\left(\sqrt{m_{u}}, \sqrt{m_{d}}, \sqrt{m_{d}}\right)$ for the neutron and $\operatorname{diag}\left(K_{P}\right)=\left(\sqrt{m_{d}}, \sqrt{m_{u}}, \sqrt{m_{u}}\right)$ for the proton, where $m_{u}$ is the "current" mass of the up quark and $m_{d}$ is the current mass of the down quark.

What is very intriguing about these $K$-matrices (which we designate with $K$ to reference Koide), is that although they originate from the thesis that baryons are magnetic monopoles, they have a form very similar to matrices which may be used in the Koide mass formula [6] for the charged leptons, namely:

$$
R=\frac{\left(\sqrt{m_{1}}+\sqrt{m_{2}}+\sqrt{m_{3}}\right)^{2}}{m_{1}+m_{2}+m_{3}} \cong \frac{3}{2} .
$$

Above, when we take $m_{1}=m_{e}, m_{2}=m_{\mu}$ and $m_{3}=m_{\tau}$ to be the charged lepton masses, the ratio $R \cong 3 / 2$ gives a very precise relationship among these masses. Indeed, if we use the 2012 PDG data

$$
\begin{aligned}
& m_{e}=0.510998928 \pm 0.000000011 \mathrm{MeV} \\
& m_{\mu}=105.6583715 \pm 0.0000035 \mathrm{MeV}
\end{aligned}
$$

and $m_{\tau}=1776.82 \pm 0.16 \mathrm{MeV}$ [7], we find using mean experimental data that $R=1.500022828$, very close to $3 / 2$.

Because the binding energies formulated in (1.2) and (1.3) are rooted in the thesis that baryons are Yang-Mills magnetic monopoles and specifically emerge from the calculation of energies via $E=-\iiint \mathfrak{L d}^{3} x$, see (11.7) of [1] et seq., and because these binding energies can also be refashioned via Koide relationships as we shall show in the next Section, the author's previous findings will provide us with the means to anchor the Koide relationships in a Lagrangian formulation. And, because Koide provides a generalization of the mass matrices derived by the author in [5], these matrices will provide us with the means to derive additional mass relationships as well, in particular, and especially, the free neutron and proton rest masses, which is the central goal of this paper.

Specifically, after reviewing in Section 2 similarities between the author's baryon/magnetic monopole matrices and the Koide matrices, we shall show in Section 3 how to reformulate the Koide relationships in terms of the statistical variance of Koide mass terms across three generations. This will yield some new Koide relationships for the neutrinos, the up quarks, and the down quarks. We then show in Section 4 how to recast these Koide relationships into a Lagrangian/energy formulation, which addresses the question as to underlying origins of these relationships, so that these relationships are not just curious coincidences, but can rooted in fundamental physics principles based on a Lagrangian.

Most importantly, in this paper, we combine the author's previous work in [1,5] as well as [8], using the generalization provided by Koide triplet mass matrices of the form (2.1) below, to deduce the observed rest masses 938.272046 MeV and 939.565379 MeV of the free neutron and proton as a function of the up and down quark masses and electric charges and the Fermi vev. This mass derivation is presented in Sections 5 and 6. In Section 7 we connect the masses obtained in Section 6 to the empirically-observed Cabibbo, Kobayashi and Maskawa (CKM) quark mixing matrices. In Section 8 we examine 
"constituent" and "vacuum-amplified" quark masses for the neutron and proton. Finally, in Section 9 we develop a Lagrangian formulation for these neutron and proton masses, which underscores that these relationships are not just close numerical coincidences, but originate from fundamental Lagrangian-based physics.

\section{Similarities between Baryon/Magnetic Monopole Matrices and Koide Matrices}

The similarities between the matrices developed by the author in [5] and those developed by Koide in [6] are highlighted if we define a Koide matrix $K_{A B}$ generally as:

$$
K_{A B} \equiv\left(\begin{array}{ccc}
\sqrt{m_{1}} & 0 & 0 \\
0 & \sqrt{m_{2}} & 0 \\
0 & 0 & \sqrt{m_{3}}
\end{array}\right) .
$$

Then, the two latent binding energy relationships (1.2) and (1.3) may be represented as:

$$
\begin{aligned}
& B_{P}=K_{A B} K_{B A}-\frac{1}{(2 \pi)^{\frac{3}{2}}} K_{A A} K_{B B}=\operatorname{Tr}\left(K^{2}\right)-\frac{1}{(2 \pi)^{\frac{3}{2}}} \operatorname{Tr}(K \otimes K) \\
& =2 m_{u}+m_{d}-\left(m_{d}+4 \sqrt{m_{u} m_{d}}+4 m_{u}\right) /(2 \pi)^{\frac{3}{2}}=7.640679 \mathrm{MeV} \\
& =\operatorname{Tr}\left(\begin{array}{ccc}
\sqrt{m_{d}} & 0 & 0 \\
0 & \sqrt{m_{u}} & 0 \\
0 & 0 & \sqrt{m_{u}}
\end{array}\right)\left(\begin{array}{ccc}
\sqrt{m_{d}} & 0 & 0 \\
0 & \sqrt{m_{u}} & 0 \\
0 & 0 & \sqrt{m_{u}}
\end{array}\right)-\frac{1}{(2 \pi)^{\frac{3}{2}}} \operatorname{Tr}\left(\begin{array}{ccc}
\sqrt{m_{d}} & 0 & 0 \\
0 & \sqrt{m_{u}} & 0 \\
0 & 0 & \sqrt{m_{u}}
\end{array}\right) \otimes\left(\begin{array}{ccc}
\sqrt{m_{d}} & 0 & 0 \\
0 & \sqrt{m_{u}} & 0 \\
0 & 0 & \sqrt{m_{u}}
\end{array}\right) \\
& B_{N}=K_{A B} K_{B A}-\frac{1}{(2 \pi)^{\frac{3}{2}}} K_{A A} K_{B B}=\operatorname{Tr}\left(K^{2}\right)-\frac{1}{(2 \pi)^{\frac{3}{2}}} \operatorname{Tr}(K \otimes K) \\
& =2 m_{d}+m_{u}-\left(m_{u}+4 \sqrt{m_{u} m_{d}}+4 m_{d}\right) /(2 \pi)^{\frac{3}{2}}=9.812358 \mathrm{MeV} \\
& =\operatorname{Tr}\left(\begin{array}{ccc}
\sqrt{m_{u}} & 0 & 0 \\
0 & \sqrt{m_{d}} & 0 \\
0 & 0 & \sqrt{m_{d}}
\end{array}\right)\left(\begin{array}{ccc}
\sqrt{m_{u}} & 0 & 0 \\
0 & \sqrt{m_{d}} & 0 \\
0 & 0 & \sqrt{m_{d}}
\end{array}\right)-\frac{1}{(2 \pi)^{\frac{3}{2}}} \operatorname{Tr}\left(\begin{array}{ccc}
\sqrt{m_{u}} & 0 & 0 \\
0 & \sqrt{m_{d}} & 0 \\
0 & 0 & \sqrt{m_{d}}
\end{array}\right) \otimes\left(\begin{array}{ccc}
\sqrt{m_{u}} & 0 & 0 \\
0 & \sqrt{m_{d}} & 0 \\
0 & 0 & \sqrt{m_{d}}
\end{array}\right)
\end{aligned}
$$

where, starting with (2.1), in (2.2) we have set $m_{1} \equiv m_{d}$ and $m_{2}=m_{3} \equiv m_{u}$ and in (2.3) we have set $m_{1} \equiv m_{u}$ and $m_{2}=m_{3} \equiv m_{d}$. Again, these originate in the author's thesis in [1] that baryons are Yang-Mills magnetic monopoles. Above, $\otimes$ designates an outer matrix pro-

duct.

On the other hand, setting $m_{1}=m_{e}, m_{2}=m_{\mu}$ and $m_{3}=m_{\tau}$ in (2.1), we may write:

$$
\operatorname{Tr}\left(K^{2}\right)=K_{A B} K_{B A}=m_{1}+m_{2}+m_{3}=m_{e}+m_{\mu}+m_{\tau}
$$

Then, using (2.4) and (2.5), Koide relationship (1.5) for charged leptons may be written as:

$$
R=\frac{\left(\sqrt{m_{e}}+\sqrt{m_{\mu}}+\sqrt{m_{\tau}}\right)^{2}}{m_{e}+m_{\mu}+m_{\tau}}=\frac{K_{A A} K_{B B}}{K_{A B} K_{B A}}=\frac{\operatorname{Tr}(K \otimes K)}{\operatorname{Tr}\left(K^{2}\right)} \cong \frac{3}{2}
$$

Clearly then, the Koide matrices (2.1) provide a general form for organizing the study of both binding energy and fermion mass relationships which lead to very accurate empirical results. It thus becomes desirable to understand the physical origin of these Koide matrices and tie them to a Lagrangian formulation so that they are no longer just intriguing curiosities that yield tantalizingly-accurate empirical results, but can also be rooted in fundamental physics principles based on a Lagrangian. And, it is desirable to see if these matrices can be extended in their application to make additional mass predictions and gain a deeper understanding of the particle 
mass spectrum, especially the free neutron and proton masses to be explored here.

We start in the next Section by showing how to reformulate the Koide relationships in terms of the statistical variance of the Koide terms across the three generations.

\section{Statistical Reformulation of the Koide Mass Relationship}

We continue to examine the charged leptons by setting $m_{1}=m_{e}, m_{2}=m_{\mu}$ and $m_{3}=m_{\tau}$ in (2.1). When we use the extremes of the experimental data ranges in [7], specifically, the largest possible tau mass and the lowest possible $m u$ mass, we obtain $R=1.5000024968$. Although this is an order of magnitude closer to $3 / 2$ than the ratio obtained from the mean data, is still outside of experimental errors. This means that while $R \cong 3 / 2$ is a very close relationship, it is still approximate even accounting for experimental error. For this to be within experimental errors, it would have to be possible to obtain some $R \leq 3 / 2$ for some combination of masses at the edges of the experimental ranges, and it is not.

First, using (2.4), we write the average of masses $\left\langle m_{i}\right\rangle$ in a Koide mass triplet $m_{1}, m_{2}, m_{3}$, i.e., the "average of the squares" of the matrix elements in (2.1), as:

$$
\begin{aligned}
\left\langle K^{2}\right\rangle & =\operatorname{Tr}\left(K^{2}\right) / 3=K_{A B} K_{B A} / 3 \\
& =\left(m_{1}+m_{2}+m_{3}\right) / 3=\left\langle m_{i}\right\rangle
\end{aligned}
$$

Next, via (2.5), we write the "square of the average" of these matrix elements as:

$$
\begin{aligned}
\langle K\rangle^{2} & =\frac{\operatorname{Tr}(K \otimes K)}{9}=\frac{K_{A A} K_{B B}}{9} \\
& =\left(\frac{\left.\sqrt{m_{1}}+\sqrt{m_{2}}+\sqrt{m_{3}}\right)^{2}}{3}\right)^{2} \\
& =\frac{\left(\sqrt{m_{1}}+\sqrt{m_{2}}+\sqrt{m_{3}}\right)^{2}}{9}
\end{aligned}
$$

So, combining (3.1) and (3.2) in the form of (1.5) allows us for the charged leptons to write:

$$
\begin{aligned}
3 \frac{\langle K\rangle^{2}}{\left\langle K^{2}\right\rangle} & =\frac{\operatorname{Tr}(K \otimes K)}{\operatorname{Tr}\left(K^{2}\right)}=\frac{K_{A A} K_{B B}}{K_{A B} K_{B A}} \\
& =\frac{\left(\sqrt{m_{1}}+\sqrt{m_{2}}+\sqrt{m_{3}}\right)^{2}}{m_{1}+m_{2}+m_{3}}=R \cong \frac{3}{2}
\end{aligned}
$$

This allows us to extract the relationship:

$$
\langle K\rangle^{2}=\frac{R}{3}\left\langle K^{2}\right\rangle \cong \frac{1}{2}\left\langle K^{2}\right\rangle,
$$

which naturally absorbs the 3 from the factor of $3 / 2$.
Now, we simply use (3.4) to form the statistical variance $\sigma(K)$ in the usual way, as:

$$
\begin{aligned}
\sigma(K) & =\left\langle K^{2}\right\rangle-\langle K\rangle^{2}=\left(1-\frac{R}{3}\right)\left\langle K^{2}\right\rangle=\left(\frac{3}{R}-1\right)\langle K\rangle^{2} \\
& =\left(\frac{3}{R}-1\right)\left\langle m_{i}\right\rangle \cong \frac{1}{2}\left\langle K^{2}\right\rangle=\langle K\rangle^{2}=\left\langle m_{i}\right\rangle .
\end{aligned}
$$

The key relationship here, using first and last terms, is:

$$
\sigma(K) \cong\left\langle m_{i}\right\rangle \text {. }
$$

So the average $\left\langle m_{i}\right\rangle$ of the charged lepton masses is approximately (and very closely) equal to the statistical variance $\sigma(K)$ of Koide matrix (2.1) when used for the charged leptons. This is a much simpler and more transparent way to express the Koide mass relationship (1.5), it completely absorbs the factor of $3 / 2$, and it is entirely equivalent to (1.5).

Of course, as noted at the outset of this Section, this is a very close, but still approximate relationship. The exact relationship, also extracted from (3.5), and using $R=1.500022828$ based on mean experimental data, is:

$$
\sigma(K)=\left(\frac{3}{R}-1\right)\left\langle m_{i}\right\rangle=0.999969563\left\langle m_{i}\right\rangle \equiv C\left\langle m_{i}\right\rangle,
$$

where we have defined the statistical coefficient $C$ and the inverse relationship for $R$ as:

$$
C \equiv \frac{3}{R}-1 ; R \equiv \frac{3}{1+C} .
$$

Thus, we may rewrite the basic Koide relationship (1.5) more generally as:

$$
\frac{\left(\sqrt{m_{1}}+\sqrt{m_{2}}+\sqrt{m_{3}}\right)^{2}}{m_{1}+m_{2}+m_{3}}=\frac{3}{1+C}=R .
$$

In the circumstance where the statistical coefficient $C$ $=1$, i.e., where the average mass is exactly equal to the statistical variance, we have $R=3 / 2$. So the statistical variance of the square roots of the three charged lepton masses is just a tiny touch less $(\times 0.999969563)$ than the average of the three masses themselves. But the factor of $3 / 2$, which is somewhat mysterious in (1.5), is now more readily understood when we realize that it corresponds with $C=1$ in (3.7).

This means that the Koide relationship for any given triplet of numbers with mass dimension $\frac{1}{2}$, may be alternatively characterized by the coefficient $C$. Thus, using (3.7), the coefficient $C$ for the charged lepton triplet is (we also include $R$ for comparison):

$$
\begin{aligned}
& C(e \mu \tau)=0.999969563 \cong 1 ; \\
& R(e \mu \tau)=1.500022828 \cong 3 / 2 .
\end{aligned}
$$


So what about some other Koide triplets? For the neutrinos, PDG in [9] provides upper limits $m_{v_{e}}<2 \mathrm{eV}$, $m_{v_{\mu}}<0.19 \mathrm{MeV}$ and $m_{v_{\tau}}<18.2 \mathrm{MeV}$ for the neutrino masses. If we use these mass limits in a Koide triplet, we find that $R=1.202960231$. But the significance of this is much more easily seen by using (3.8) to calculate:

$$
\begin{aligned}
& C\left(v_{e} v_{\mu} v_{\tau}\right)=1.4938480 \cong 3 / 2 ; \\
& R\left(v_{e} v_{\mu} v_{\tau}\right)=1.20296023 \cong 6 / 5
\end{aligned}
$$

Here, we have another ratio very close to $3 / 2$, but now it is the coefficient $C$ rather than the coefficient $R$. So, for the upper neutrino mass limits, $\sigma\left(K_{v}\right) \cong(3 / 2)\left\langle m_{v}\right\rangle$. This in an interesting "coefficient migration" as between the charged and uncharged leptons, wherein for the charged leptons masses $R \cong 3 / 2$ to parts per 100,000 , while for the neutrino lepton upper mass limits, $C \cong 3 / 2$ within about $0.4 \%$. As we shall see, this is the start of a new Koide pattern.

Turning to quark masses, we use $m_{u}=2.223792405$ $\mathrm{MeV}$ and $m_{d}=4.906470335 \mathrm{MeV}$ developed in (10.3) and (10.4) of [5] with the conversion $1 \mathrm{u}=931.494061(21)$ $\mathrm{MeV} / \mathrm{c}^{2}$. We also use $m_{c}=1.275 \pm 0.025 \mathrm{GeV}, m_{s}=95$ $\pm 5 \mathrm{MeV}, \quad m_{t}=173.5 \pm .6 \pm .8 \mathrm{GeV}$ and $m_{b}=4.18 \pm$ $0.03 \mathrm{GeV}$ from PDG's [10]. For Koide triplets of a single electric charge type, we can then calculate that:

$$
\begin{aligned}
& C(u c t)=1.54688 \cong 3 / 2 ; \\
& R(u c t)=1.177913486 \cong 6 / 5 \\
& C(d s b)=1.18741 \cong 6 / 5 ; \\
& R(d s b)=1.371483911 \cong 15 / 11
\end{aligned}
$$

So we now see a distinctive pattern of coefficient migration among (3.10) through (3.13). For the charged leptons in (3.10) which are the lower members of a weak isospin doublet, $R(e \mu \tau) \cong 3 / 2$, as has long been known. For neutrinos which are the upper members of this doublet, $C\left(v_{e} v_{\mu} v_{\tau}\right) \cong 3 / 2$, which migrates the $3 / 2$ from the $R$ to the $C$ coefficient. Then, for the up quarks, we find another coefficient migration such that $C(u c t) \cong 3 / 2$, which is same as the $C$ for the neutrinos. Both the up quarks and the neutrinos are the upper members of weak isospin doublets. Finally, we see that the $R(u c t) \cong 6 / 5$ coefficient for the up quarks, now migrates to $C(d s b)$ $\cong 6 / 5$ for down quarks.

So the migration is $R(e \mu \tau) \cong 3 / 2 \rightarrow C\left(v_{e} v_{\mu} v_{\tau}\right) \cong 3 / 2$ for leptons, $C\left(v_{e} v_{\mu} v_{\tau}\right) \cong 3 / 2 \rightarrow C(u c t) \cong 3 / 2$ providing a "bridge" from "up" leptons to "up" quarks, and then $R(u c t) \cong 6 / 5 \rightarrow C(d s b) \cong 6 / 5$ migrating from the up to the down quarks.

The net upshot of this coefficient migration is that we now have Koide-style close relations for all four sets of fermions (and anti-fermions) of like-electric charge $Q$, namely:

$$
\begin{aligned}
& R(Q=0)=\frac{\left(\sqrt{m_{v(e)}}+\sqrt{m_{v(\mu)}}+\sqrt{m_{v(\tau)}}\right)^{2}}{m_{v(e)}+m_{v(\mu)}+m_{v(\tau)}} \cong \frac{6}{5} \\
& R(Q= \pm 1)=\frac{\left(\sqrt{m_{e}}+\sqrt{m_{\mu}}+\sqrt{m_{\tau}}\right)^{2}}{m_{e}+m_{\mu}+m_{\tau}} \cong \frac{3}{2} \\
& R\left(Q= \pm \frac{2}{3}\right)=\frac{\left(\sqrt{m_{u}}+\sqrt{m_{c}}+\sqrt{m_{t}}\right)^{2}}{m_{u}+m_{c}+m_{t}} \cong \frac{6}{5} \\
& R\left(Q= \pm \frac{1}{3}\right)=\frac{\left(\sqrt{m_{d}}+\sqrt{m_{s}}+\sqrt{m_{b}}\right)^{2}}{m_{d}+m_{s}+m_{b}} \cong \frac{15}{11}
\end{aligned}
$$

Each of these relationships takes twelve a priori independent fermion masses and reduces by 1 , their mutual independence. So with (3.14) through (3.17), to first approximation, we have now eight, rather than twelve independent fermion masses.

For some other commonly-studied Koide triplets we have:

$$
\begin{aligned}
& C(u d s)=0.69290 \cong 1 / \sqrt{2} \\
& R(u d s)=1.772105341 \cong \frac{3 \sqrt{2}}{1+\sqrt{2}} \\
& C(c t b)=1.00939 \cong 1 ; \\
& R(c t b)=1.492994103 \cong 3 / 2 \\
& C(u s c)=0.86795 ; R(u s c)=1.606042302 \\
& C(c s b)=1.02783 \cong 1 ; \\
& R(c s b)=1.479416975 \cong 3 / 2\left(\text { with }-\sqrt{m_{s}}\right) \\
& C(d c s)=0.81520 ; R(d c s)=1.652718083 .
\end{aligned}
$$

We note that the relationship (3.18) for $C(u d s)$ $\cong 1 / \sqrt{2}$ is accurate to within experimental errors. Specifically, given the empirical $m_{s}=95 \pm 5 \mathrm{MeV}$, (3.18) can be made into an exact relationship to ten digits (the accuracy of the up and down masses derived in [5]) if we set $m_{s}=98.95303495 \mathrm{MeV}$. Of course, even the relationship (3.15) for the charged leptons is a close but not exact relationship, see the discussion at the start of this Section, so we ought not expect (3.18) to be exactly $C(u d s)=1 / \sqrt{2}$. But, similarly to (1.5), see also (3.10), it may well make sense to regard this as a relationship accurate to the first three or four decimal places, which would improve our knowledge of the strange quark mass by four or five orders of magnitude.

But this main point of the foregoing is not about the specific Koide relationships (though the set of relationships (3.14), (3.16) and (3.17) are important steps for- 
ward in their own right), but about how the ratio parameter $R$ which for the charged lepton triplet is $R \cong 3 / 2$, can be reformulated for any fermion triplet into the coefficient $C$ in the statistical variance relationship $\sigma(K)=C\left\langle m_{i}\right\rangle$, which, for the charged leptons, is $C \cong 1$. And, as we see in (3.14) through (3.17), this can lead to additional rela- tionships via a cascading migration of coefficients.

Turning back to the neutron and proton triplets,

$$
\begin{aligned}
& \operatorname{diag}\left(K_{P}\right)=\left(\sqrt{m_{d}}, \sqrt{m_{u}}, \sqrt{m_{u}}\right), \\
& \operatorname{diag}\left(K_{N}\right)=\left(\sqrt{m_{u}}, \sqrt{m_{d}}, \sqrt{m_{d}}\right)
\end{aligned}
$$

which were so central to obtaining accurate binding energy predictions in $[1,5]$, we find using the $\mathrm{MeV}$ equivalents of the mass values $m_{u}=2.223792405 \mathrm{MeV}$, $m_{d}=4.906470335 \mathrm{MeV}$ obtained in (10.3) and (10.4) of [5] that:

$$
\begin{aligned}
& C(p=d u u)=0.0387876019 \\
& R(p=d u u)=2.8879821000 \\
& C(n=u d d)=0.0298844997 \\
& R(n=u d d)=2.9129480061
\end{aligned}
$$

For these triplets which all have a small variance in comparison to the earlier triplets which cross generations, the Koide ratio $R \cong 3$. In the circumstance where the variance is exactly zero because all three quarks have the same mass, for example, for the triplets $\Delta^{++}=u u u$ and $\Delta^{-}=d d d$, using the Koide mass relationship for parameterization, we have $C=0 ; R=3$.

\section{Lagrangian/Energy Reformulation of the Koide Mass Relationship}

The appearance of Koide triplets originating from the thesis that Baryons are Yang-Mills magnetic monopoles can be seen, for example, by considering Equation (11.2) of [1] for the field strength tensor of a Yang-Mills magnetic monopole containing a triplet of colored quarks in the zero-perturbation limit, reproduced below:

$$
\begin{aligned}
& \operatorname{Tr} F^{\mu v}=-i\left(\frac{\bar{\psi}_{R}\left[\gamma^{\mu}{ }^{\mu} \gamma^{v}\right] \psi_{R}}{" p_{R}-m_{R} "}\right. \\
& \left.+\frac{\bar{\psi}_{G}\left[\gamma^{\mu}{ } \gamma^{v}\right] \psi_{G}}{" p_{G}-m_{G} "}+\frac{\bar{\psi}_{B}\left[\gamma^{\mu}{ }_{v} \gamma^{v}\right] \psi_{B}}{" p_{B}-m_{B} "}\right) \\
& E_{\otimes}=-\iiint \mathfrak{L}_{\otimes} \mathrm{d}^{3} x=\frac{1}{2} \operatorname{Tr} \iiint F_{\mu \nu} \otimes F^{\mu v} \mathrm{~d}^{3} x=\frac{1}{2} \operatorname{Tr} \iiint F_{A B} \cdot F_{C D} \mathrm{~d}^{3} x=\frac{1}{2} \iiint F_{A A} \cdot F_{B B} \mathrm{~d}^{3} x=\frac{1}{(2 \pi)^{\frac{3}{2}}} K_{A A} K_{B B} \\
& =\frac{1}{(2 \pi)^{\frac{3}{2}}} \operatorname{Tr}\left[\left(\begin{array}{ccc}
\sqrt{m_{1}} & 0 & 0 \\
0 & \sqrt{m_{2}} & 0 \\
0 & 0 & \sqrt{m_{3}}
\end{array}\right) \otimes\left(\begin{array}{ccc}
\sqrt{m_{1}} & 0 & 0 \\
0 & \sqrt{m_{2}} & 0 \\
0 & 0 & \sqrt{m_{3}}
\end{array}\right)\right]=\frac{1}{(2 \pi)^{\frac{3}{2}}}\left(\sqrt{m_{1}}+\sqrt{m_{2}}+\sqrt{m_{3}}\right)^{2}
\end{aligned}
$$

If we generalize this to any three fermion wavefunctions $\psi_{1}, \psi_{2}, \psi_{3}$ such that (4.1) represents the specific case $\psi_{1}=\psi_{R}, \psi_{2}=\psi_{G}$ and $\psi_{3}=\psi_{B}$, and, as we did prior to (11.19) of [1], if we consider the circumstance in which the interactions shown in Figure 1 at the start of Section 3 in [1] occur essentially at a point, then $\left[\gamma^{\mu}{ }_{v} \gamma^{v}\right] \rightarrow\left[\gamma^{\mu}, \gamma^{v}\right]$ approaches an ordinary commutator, each of the $p \rightarrow 0$, and the "quoted" denominator becomes an ordinary denominator, see (3.9) through (3.12) of [1] for further background. So also setting $m_{1}=m_{R}, m_{2}=m_{G}$ and $m_{3}=m_{B}$, (4.1) generalizes for a point interaction to a Koide-style field strength tensor:

$$
\begin{aligned}
\operatorname{Tr} F^{\mu v} & =-i\left(\frac{\bar{\psi}_{1}\left[\gamma^{\mu}, \gamma^{v}\right] \psi_{1}}{m_{1}}\right. \\
& \left.+\frac{\bar{\psi}_{2}\left[\gamma^{\mu}, \gamma^{v}\right] \psi_{2}}{m_{2}}+\frac{\bar{\psi}_{3}\left[\gamma^{\mu}, \gamma^{v}\right] \psi_{3}}{m_{3}}\right)
\end{aligned}
$$

Then, we form a pure gauge field Lagrangian

$$
\mathfrak{\varrho}_{\text {gauge }}=-\frac{1}{2} \operatorname{Tr}\left(F_{\mu v} F^{\mu v}\right)=-\frac{1}{2} \operatorname{Tr}(F \cdot F)
$$

as in (11.7) of [1]. As discussed in Section 3 of [5], we consider both inner and outer products over the YangMills indexes of $F$, i.e., we consider both $\operatorname{Tr} F^{2}=$ $\operatorname{Tr}\left(F_{A B} \cdot F_{B C}\right)=F_{A B} \cdot F_{B A}$ and $\operatorname{Tr}(F \otimes F)=\operatorname{Tr}\left(F_{A B} \cdot F_{C D}\right)$ $=F_{A A} \cdot F_{B B}$. Note carefully the different index structures in $F_{A B} \cdot F_{B A}$ versus $F_{A A} \cdot F_{B B}$, and also contrast this to (2.2) through (2.5) in this paper, which we shall now seek to refashion into a Lagrangian formulation.

To proceed, we use this Lagrangian $\mathfrak{L}_{\text {gauge }}$ to calculate energies according to (11.7) of [1], also (1.8) of [5], which are reproduced below:

$$
E=-\iiint \mathfrak{L}_{\text {gauge }} \mathrm{d}^{3} x=\frac{1}{2} \operatorname{Tr} \iiint F_{\mu v} F^{\mu v} \mathrm{~d}^{3} x .
$$

In the case where $\psi_{1}=\psi_{d}, \psi_{2}=\psi_{3}=\psi_{u}$ so that $F^{\mu v}=F_{P}^{\mu v}$ represents the proton, then depending on whether we contact indexes using $F_{A B} \cdot F_{B A}$ or 
while the Koide generalization of the inner products ( $K_{A B} K_{B A}$ index summation) is:

$$
\begin{aligned}
E & =-\iiint \mathcal{L d}^{3} x=\frac{1}{2} \operatorname{Tr} \iiint F_{\mu \nu} F^{\mu \nu} \mathrm{d}^{3} x=\frac{1}{2} \operatorname{Tr} \iiint F_{A B} \cdot F_{B D} \mathrm{~d}^{3} x=\frac{1}{2} \iiint F_{A B} \cdot F_{B A} \mathrm{~d}^{3} x \\
& =\frac{1}{(2 \pi)^{\frac{3}{2}}} K_{A B} K_{B A}=\frac{1}{(2 \pi)^{\frac{3}{2}}} \operatorname{Tr}\left[\left(\begin{array}{ccc}
\sqrt{m_{1}} & 0 & 0 \\
0 & \sqrt{m_{2}} & 0 \\
0 & 0 & \sqrt{m_{3}}
\end{array}\right)\left(\begin{array}{ccc}
\sqrt{m_{1}} & 0 & 0 \\
0 & \sqrt{m_{2}} & 0 \\
0 & 0 & \sqrt{m_{3}}
\end{array}\right)\right]=\frac{1}{(2 \pi)^{\frac{3}{2}}}\left(m_{1}+m_{2}+m_{3}\right)
\end{aligned}
$$

This means that is now becomes possible to express the Koide relationship (3.9) entirely in terms of energies $E$ derived from the Lagrangian integration (4.3). Specifically, combining (3.9) with (4.4) and (4.5) allows us to write:

$$
\begin{aligned}
\frac{E_{\otimes}}{E} & =\frac{\iiint \mathfrak{L}_{\otimes} \mathrm{d}^{3} x}{\iiint \mathfrak{Q}^{3} x}=\frac{\operatorname{Tr} \iiint F_{\mu \nu} \otimes F^{\mu v} \mathrm{~d}^{3} x}{\operatorname{Tr} \iiint F_{\mu v} F^{\mu v} \mathrm{~d}^{3} x} \\
& =\frac{\operatorname{Tr} \iiint F \otimes F \mathrm{~d}^{3} x}{\operatorname{Tr} \iiint F^{2} \mathrm{~d}^{3} x} \\
& =\frac{\iiint F_{A A} \cdot F_{B B} \mathrm{~d}^{3} x}{\iiint F_{A B} \cdot F_{B A} \mathrm{~d}^{3} x}=\frac{K_{A A} K_{B B}}{K_{A B} \cdot K_{B A}} \\
& =\frac{\left(\sqrt{m_{1}}+\sqrt{m_{2}}+\sqrt{m_{3}}\right)^{2}}{m_{1}+m_{2}+m_{3}}=\frac{3}{1+C}=R
\end{aligned}
$$

This expresses the Koide mass relationship in multiple forms, in terms of an energy integral of the general Lagrangian density form

$$
\mathfrak{L}=-\frac{1}{2} \operatorname{Tr}(F \cdot F),
$$

with general field strength (4.2). This means for any Koide triplet of given empirical $R$, there is an energy $E_{R}$ which vanishes under condition:

$$
\begin{aligned}
E_{R} & =\iiint\left(\mathfrak{E}_{\otimes}-R \mathfrak{Q}\right) \mathrm{d}^{3} x \\
& =\operatorname{Tr} \iiint\left(F \otimes F-R F^{2}\right) \mathrm{d}^{3} x=0
\end{aligned}
$$

This is the Lagrangian/energy formulation of the Koide relationship (3.9), and although different in appearance, it is entirely equivalent. So, for example, using the symbol $\therefore$ as in Figure 1 and Table 3 of [8] to represent the three generations of the fermions for any given charge, the four Koide relationships (3.14) through (3.17) for the pole (low probe energy) masses may be written as in the entirely equivalent, alternative form:

$$
\begin{aligned}
E_{v: .} & =\iiint\left(\mathfrak{L}_{\otimes}-\frac{6}{5} \mathfrak{\&}\right) \mathrm{d}^{3} x \\
& =\operatorname{Tr} \iiint\left(F \otimes F-\frac{6}{5} F^{2}\right) \mathrm{d}^{3} x \cong 0
\end{aligned}
$$

$$
\begin{aligned}
E_{e::} & =\iiint\left(\mathfrak{L}_{\otimes}-\frac{3}{2} \mathfrak{L}\right) \mathrm{d}^{3} x \\
& =\operatorname{Tr} \iiint\left(F \otimes F-\frac{3}{2} F^{2}\right) \mathrm{d}^{3} x \cong 0 \\
E_{u::} & =\iiint\left(\mathfrak{L}_{\otimes}-\frac{6}{5} \mathfrak{L}\right) \mathrm{d}^{3} x \\
& =\operatorname{Tr} \iiint\left(F \otimes F-\frac{6}{5} F^{2}\right) \mathrm{d}^{3} x \cong 0 \\
E_{d::} & =\iiint\left(\mathfrak{L}_{\otimes}-\frac{15}{11} \mathfrak{L}\right) \mathrm{d}^{3} x \\
& =\operatorname{Tr} \iiint\left(F \otimes F-\frac{15}{11} F^{2}\right) \mathrm{d}^{3} x \cong 0
\end{aligned}
$$

Whether these become exactly equal to zero for masses at high-probe energies, and whether there is an underlying action principle involved here, are questions beyond the scope of this paper which are worth consideration.

What ties all of this together, is that we model the radial behavior of each fermion in the triplet $\psi_{1}, \psi_{2}, \psi_{3}$ using the Gaussian ansatz borrowed from Equation (14) of [3] and introduced in (9.9) of [1] which is reproduced below with an added label $i=1,2,3$ for each of the fermions and masses in (4.2):

$$
\psi_{i}(r)=u_{i}(p)\left(\pi \lambda_{i}^{2}\right)^{-\frac{3}{4}} \exp \left(-\frac{1}{2} \frac{\left(r-r_{0 i}\right)^{2}}{\lambda_{i}^{2}}\right),
$$

and that we also relate each reduced Compton wavelength $\lambda_{i}$ to its corresponding mass $m_{i}$ via the DeBroglie relation $\lambda_{i}=\hbar / m_{i} c$, see [1] following (11.18). This is what makes it possible to precisely, analytically calculate the energy in integrals of the form (4.3), specifically making use of the mathematical Gaussian relationship (9.11) of [1]:

$$
\iiint \frac{1}{\pi^{\frac{3}{2}} \lambda^{3}} \exp \left(-\frac{\left(r-r_{0}\right)^{2}}{\lambda^{2}}\right) \mathrm{d}^{3} x=1,
$$

and variants thereof. It is (4.12) and (4.13) and $\lambda_{i}=1 / m_{i} \quad$ (in $\hbar=c=1$ units) which tie everything together at the "nuts and bolts" mathematical level when 
(4.2) is employed in (4.3) through (4.11). And this is what leads to accurate mass relationship (1.1) and binding energy predictions (1.2) and (1.3), as well as the binding energy predictions for ${ }^{2} \mathrm{H},{ }^{3} \mathrm{H},{ }^{3} \mathrm{He}$ and ${ }^{4} \mathrm{He}$ and the proton-neutron mass difference (1.4) found in [5].

The final piece which also ties this together at nuts and bolts level, is the empirical normalization for fermion wavefunctions developed in (11.30) of [1], namely:

$$
N^{4}=\frac{1}{n_{f}} \frac{(E+m)^{2}}{(2 m)^{2}}=\frac{1}{24} \frac{(E+m)^{2}}{(2 m)^{2}},
$$

where $n_{f}=24$ is the total number of fermions over three generations including three colors for each quark.

Now, it is important to emphasize that the Gaussian ansatz (4.12) is not a theory, but rather, it is a modeling hypothesis that allows us to analytically perform the necessary integrations and calculate energies which fortuitously turn out to correlate very well with empirical data. That is, explicitly in [1] and implicitly in [5], we hypothesized that the fermion wavefunctions can be modeled as Gaussians with specific Compton wavelengths $\lambda_{i}=1 / m_{i}$ defined to match the current quark masses, we performed the integrations in (4.3), and we found that the energies predicted matched empirical binding data to - in most cases_-parts per million. This, in turn, tells us that for the purpose of predicting binding energies, it is possible to model the current quarks as Gaussians (which means they act as free fermions), with masses and wavelengths based on their undressed, current quark masses, and to thereby obtain empiricallyvalidated results.

But, as also discussed at the end of Section 11 in [1], this use of a current quark mass does not apply when it comes predicting the short range of the nuclear interaction which we showed at the end of Section 10 in [1] is indeed short range with a standard deviation of $\sigma=1 / \sqrt{2} \lambda$. For, if we use the current quark masses that work so well for binding energies, we find $\lambda_{u} \sim 85.65 F$ and $\lambda_{d} \sim 41.04 F$, and the predicted short range is still not short enough. If, however, we turn to the constituent quark masses which, at the end of Section 11 in [1], for estimation, we took to be $939 \mathrm{MeV} / 3=313 \mathrm{MeV}$, then we have $\lambda \sim 0.63 F$ and $\sigma=\frac{1}{\sqrt{2}} \lambda \sim 0.45 F$, which tells us that the nuclear interaction virtually ceases at about $4 \sigma \approx 3 \lambda \sim 2 F$. This is exactly what is observed.

In both cases - for nuclear binding energies and for the nuclear interaction short range - we found that the Gaussian ansatz (4.12) does yield empirically-accurate results. But for binding energies, it was the undressed, current quark masses which gave us the right results, while for nuclear short range, it was the fully dressed, constituent quarks masses that were needed to obtain the correct re- sult.

Because we shall momentarily embark on a prediction of the fully dressed rest masses 938.272046 MeV and $939.565379 \mathrm{MeV}$ of the free neutron and free proton, what we learn from this is that while we might also be able to approach the neutron and proton masses using a Gaussian ansatz for fermion wavefunctions, we will, however, need to be judicious in the fermion wavefunctions we choose and in the masses that we assign to the fermions. That is, the focus of our deliberations will be, not whether we can use the Gaussian ansatz, but on how to select the fermion wavefunctions and masses that we do use with the Gaussian ansatz, in order to obtain empirically accurate results.

Now, with all of the foregoing as background, let us see how to predict the neutron and proton masses.

\section{Predicting the Neutron plus Proton Mass Sum to within about 6 Parts in 10,000}

Because we can connect any Koide matrix products to a Lagrangian via (4.4) and (4.5), let us work directly with the Koide matrix (2.1) to determine how to assign the masses $m_{1}, m_{2}, m_{3}$ so as to predict the neutron and proton masses. Then at the end (in Section 9), we can backtrack using the development in Section 4 to connect these masses to their associated Lagrangian. In other words, we will first fit the empirical mass data, then we will backtrack to the underlying Lagrangian.

Each of the neutron and proton contains three quarks. The sum of the current quark masses is $2 m_{d}+m_{u}=$ $12.0367331 \mathrm{MeV}$ for the neutron and $2 m_{u}+m_{d}=$ 9.35405514 MeV for the proton, using

$m_{u}=2.223792405 \mathrm{MeV}$ and $m_{d}=4.906470335 \mathrm{MeV}$ earlier introduced before (3.12) as developed in (10.3) and (10.4) of [5]. For a free neutron and proton, none of this rest mass is released as binding energy, and so these quark mass sums are fully included in

$M_{N}=939.565379 \mathrm{MeV}$ and $M_{P}=938.272046 \mathrm{MeV}$ respectively, where we use an uppercase $M$ to denote these fully-dressed, observed masses. As demonstrated in Sections 11 and 12 of [1] and throughout [5], these rest masses are reduced when the neutron and proton fuse with other nucleons. But for free protons and neutrons, the entire rest mass is retained and all of the latent binding energy is used to confine quarks.

This means the "mass coverings" $m$ (using a lowercase $m$ ) for the neutron and proton may be calculated to be:

$$
\begin{aligned}
& m_{P} \equiv M_{P}-2 m_{u}-m_{d}=928.9179915 \mathrm{MeV}, \\
& m_{N} \equiv M_{N}-2 m_{u}-m_{d}=927.5286457 \mathrm{MeV} .
\end{aligned}
$$

These mass coverings $m$ represent the observed, fully-dressed neutron and proton masses $M$, less the sum $K_{A B} K_{B A}=m_{1}+m_{2}+m_{3}$ of the current quark masses, 
with $m_{1} \equiv m_{d}, m_{2}=m_{3} \equiv m_{u}$ for the proton, and $m_{1} \equiv m_{u}, m_{2}=m_{3} \equiv m_{d}$ for the neutron, see (2.4). One may think of $m_{P}$ and $m_{N}$ as weights of rather heavy "clothing" "covering" "bare" quarks. The sum of these two mass covers is:

$$
\begin{aligned}
m_{N}+m_{P} & =M_{N}+M_{P}-3 m_{u}-3 m_{d} \\
& =1856.446637 \mathrm{MeV}
\end{aligned}
$$

Now, at the end of Section 10 of [5], after deriving the neutron minus proton mass difference (1.4), we noted that the individual masses for the neutron and proton could now be obtained by deriving some independent expression related to the sum of their masses, and then solving these two simultaneous equations - sum equation and difference equation-for the two target masses, namely, those of the neutron and proton. We shall do exactly that here. In particular, it will be our goal to derive the sum $M_{N}+M_{P}$ of these two masses, and then use (1.4) as a simultaneous equation to obtain each separate mass. The benefit of this approach using a sum, referring to the so-called mass "toolbox" in (4.11) of [5] and also the discussion of the alpha nuclide following (5.4) of [5], is that in selecting mass terms to consider, we can eliminate any candidates not absolutely symmetric under $p \leftrightarrow n$ and $u \leftrightarrow d$ interchange, because the sum $M_{N}+M_{P}$ contains three up quarks and three down quarks, as well as one neutron and one proton. Our empirical target, therefore is the mass sum $M_{N}+M_{P}=$ $1877.837425 \mathrm{MeV}$ But we can alternatively find this by finding the mass cover sum $m_{P}+m_{N}=1856.446637$ $\mathrm{MeV}$ of (5.3) to which we can then readily add $3 m_{u}+3 m_{d}$. These sums are what we now seek to predict.

We now return to use the "clues" laid out in (3.6) through (3.8) of [8]. We start in the simplest way possible by focusing our consideration on (3.8) of [8], reproduced below, but multiplied by a factor of 2 and separated into $\sqrt[4]{v_{F} m_{u}}$ and $\sqrt[4]{v_{F} m_{d}}$ in the second term, thus:

$$
\begin{aligned}
2 \sqrt{v_{F} \cdot \sqrt{m_{u} m_{d}}} & =2 \sqrt[4]{v_{F} m_{u}} \sqrt[4]{v_{F} m_{d}}=2 \sqrt[4]{v_{F}^{2} m_{u} d_{d}} \\
& =1803.670518 \mathrm{MeV}
\end{aligned}
$$

Here, $v_{F}=246.219651 \mathrm{GeV}$ is the Fermi vev. Because this is about $3 \%$ smaller than $m_{P}+m_{N}$ in (5.3) and is closer to $m_{P}+m_{N}$ than either (3.6) or (3.7) of [8], and also is symmetric under $u \leftrightarrow d$ interchange, we shall see if (5.4) can be used, by itself, to provide the foundation for hitting the $m_{P}+m_{N}=1856.446637 \mathrm{MeV}$ mass target (5.3). As we shall, it can be so used!

In (4.11) of [5], we developed a "toolkit" of masses which we used for calculating the binding and fusion release energies of all the 1s nuclides with very close precision. We shall wish to add to this toolkit here, and in particular, will wish to refine our use of the Fermi vev $v_{F}$ $=246.219651 \mathrm{GeV}$ beyond what is shown in (5.4). Specifically, as noted after (3.8) of [8], we need to put (5.4) "and like expressions into the right context and obtain the right coefficients. And where do such coefficients come from? The generators of a GUT!"

Now it is time to "cash in" on the GUT we developed in [8] to obtain the coefficients needed to bring (5.4) closer to the target mass of $1856.446637 \mathrm{MeV}$ in (5.3). Because the vev that seems based on (5.4) to bring us into the correct "ballpark" is the Fermi vev, we focus on electroweak symmetry breaking which occurs at the Fermi vev, and which, in (8.2) of [8], is specified by breaking electroweak symmetry using electric charge generator $Q$ via:

$$
\begin{aligned}
& \operatorname{diag}\left(\Phi_{F}\right)=\operatorname{diag}\left(T^{i} \varphi_{i F}\right) \\
& \equiv v_{F}\left(0, \frac{2}{3},-\frac{1}{3},-\frac{1}{3},-1,-\frac{1}{3}, \frac{2}{3}, \frac{2}{3}\right)=v_{F} \operatorname{diag} Q
\end{aligned}
$$

For the proton with a fermion triplet $(d, u, u)$, the corresponding eigenvalue entries in (5.5) above are

$$
\left(-\frac{1}{3} v_{F}, \frac{2}{3} v_{F}, \frac{2}{3} v_{F}\right)
$$

For the neutron and its $(u, d, d)$ triplet, the entries are

$$
\left(\frac{2}{3} v_{F},-\frac{1}{3} v_{F},-\frac{1}{3} v_{F}\right) \text {. }
$$

We now wish to use these to establish Koide triplet matrices for the neutron and proton which can then be used to generate the sum of their masses.

Looking at these vacuum triplets

$$
\left(-\frac{1}{3} v_{F}, \frac{2}{3} v_{F}, \frac{2}{3} v_{F}\right)
$$

and

$$
\left(\frac{2}{3} v_{F},-\frac{1}{3} v_{F},-\frac{1}{3} v_{F}\right),
$$

we see that to match the mass dimension $\frac{1}{2}$ of the terms with $\sqrt[4]{v m_{u}}$ and $\sqrt[4]{v m_{d}}$ in (5.4) and use these as Koide triplets, we will need to take the fourth roots of these vacuum triplets. So we do exactly that, and pair these triplets with the mass triplets $\left(m_{d}, m_{u}, m_{u}\right)$ and $\left(m_{u}, m_{d}, m_{d}\right)$ for which we also take the fourth root to match (5.4). Thus, we use

$$
\left(-\frac{1}{3} v_{F}, \frac{2}{3} v_{F}, \frac{2}{3} v_{F}\right) \rightarrow\left(i \sqrt[4]{\frac{1}{3} v_{F} m_{d}}, \sqrt[4]{\frac{2}{3} v_{F} m_{u}}, \sqrt[4]{\frac{2}{3} v_{F} m_{u}}\right)
$$


and

$$
\left(\frac{2}{3} v_{F},-\frac{1}{3} v_{F},-\frac{1}{3} v_{F}\right) \rightarrow\left(\sqrt[4]{\frac{2}{3} v_{F} m_{u}}, i^{0.5} \sqrt[4]{\frac{1}{3} v_{F} m_{d}}, i \sqrt[0.5]{\frac{1}{3} v_{F} m_{d}}\right)
$$

to define two new Koide triplets, one for the neutron and one for the proton, as follows:

$$
\begin{gathered}
K_{A B}(N) \equiv\left(\begin{array}{ccc}
\sqrt[4]{\frac{2}{3} v_{F} m_{u}} & 0 & 0 \\
0 & i \sqrt{0.5} \sqrt[4]{\frac{1}{3} v_{F} m_{d}} & 0 \\
0 & 0 & i_{A B}^{0.5} \sqrt[4]{\frac{1}{3} v_{F} m_{d}}
\end{array}\right) \\
K_{i}(P) \equiv\left(\begin{array}{ccc}
i^{0.5} \sqrt[4]{\frac{1}{3} v_{F} m_{d}} & 0 & 0 \\
0 & \sqrt[4]{\frac{2}{3} v_{F} m_{u}} & 0 \\
0 & 0 & \sqrt[4]{\frac{2}{3} v_{F} m_{u}}
\end{array}\right)
\end{gathered}
$$

What we have done here is simply develop (5.6) and (5.7) to match the mass dimensionalities in (5.4) while bringing in the coefficients from (5.5) which reflect the electric charges of the up and down quarks. We see that because of the negatively-signed (-) charge for the down quark, of which we have taken the fourth root, each of these triplets contains components with the complex coefficient

$$
\sqrt[4]{-1}=i^{0.5}=\frac{1}{\sqrt{2}}(1+i)
$$

In recent years, consideration has been given to having negative square root terms in Koide mass relations, see for example (3.21) in which one uses $-\sqrt{m_{s}}$ to derive a close relation for the $(c s b)$ triplet (see Rivero's original finding of this in [11]). The above, (5.6) and (5.7) take this a step further, because they raise the specter of Koide triplets with complex square root coefficients! In the next Section we explore the profound implications of these complex coefficients, which arise from the oppositelysigned charges of the up and down quarks. But for the moment, we ignore $i^{0.5}$ in the above and examine magnitudes only, and form and calculate the following Koide matrix product from (5.6) and (5.7) with $i^{0.5}$ excised:

$$
\begin{aligned}
& K_{A B}(P) K_{B A}(N)=\operatorname{Tr}\left[\left(\begin{array}{ccc}
\sqrt[4]{\frac{1}{3} v_{F} m_{d}} & 0 & 0 \\
0 & \sqrt[4]{\frac{2}{3} v_{F} m_{u}} & 0 \\
0 & 0 & \sqrt[4]{\frac{2}{3} v_{F} m_{u}}
\end{array}\right) \times\left(\begin{array}{ccc}
\sqrt[4]{\frac{2}{3} v_{F} m_{u}} & 0 & 0 \\
0 & \sqrt[4]{\frac{1}{3} v_{F} m_{d}} & 0 \\
0 & 0 & \sqrt[4]{\frac{1}{3} v_{F} m_{d}}
\end{array}\right)\right] \\
& =3 \cdot \sqrt[4]{\frac{2}{9} v_{F}^{2} m_{u} m_{d}}=1857.570635 \mathrm{MeV}
\end{aligned}
$$

Comparing to (5.3) which tells us that

$$
\left(m_{P}+m_{N}\right)_{\text {Observed }}=1856.446637 \mathrm{MeV}
$$

we see that we have hit the target to within about $0.06 \%$ ! That is:

$$
\begin{aligned}
& \qquad \begin{array}{l}
\frac{K_{A B}(P) K_{B A}(N)}{\left(m_{N}+m_{P}\right)_{\text {Observed }}}=\frac{1857.570635 \mathrm{MeV}}{1856.446637 \mathrm{MeV}} \\
=1.000605457 !
\end{array} \\
& \qquad \begin{array}{l}
M_{N}+M_{P}=m_{N}+m_{P}+3 m_{u}+3 m_{d} \cong K_{A B}(P) K_{B A} \\
\text { So it appears as though we have now discovered the }
\end{array} \\
& \text { correct coefficients for the "clue" in (5.4). These coeffi- } \\
& \text { cients, which are based on none other than the electric }
\end{aligned}
$$

$$
M_{N}+M_{P}=m_{N}+m_{P}+3 m_{u}+3 m_{d} \cong K_{A B}(P) K_{B A}(N)+3 m_{u}+3 m_{d}=3 \cdot \sqrt[4]{\frac{2}{9} v_{F}^{2} m_{u} m_{d}}+3 m_{u}+3 m_{d} .
$$

This is extremely close, and in particular, we now see that the sum of the neutron and proton mass coverings may be expressed solely as a function of the up and down quark masses and charges and the Fermi vev to within about 6 parts in 10,000! So if we use this close relationship to hypothesize that a meaningful relationship is given by $m_{N}+m_{P} \cong K_{A B}(P) K_{B A}(N)$, then using the above with (5.3) to add the current quark masses $3 m_{u}+3 m_{d}$ to this mass cover sum, we see that to within about $0.06 \%$ : charges of the quarks, yield the neutron plus proton mass sum to 6 parts in 10,000 !

Further qualifying (5.10) as a proper and not merely 
coincidental expression for the neutron plus proton mass sum, we see that this is symmetric under $u \leftrightarrow d$ interchange, and that it is formed by taking the inner product $K_{A B}(P) K_{B A}(N)$ of the Koide proton matrix $K(P)$ and the Koide neutron matrix $K(N)$, which product is symmetric under $p \leftrightarrow n$ interchange. Further, both of these fully embed the electric charges and mass magnitudes of the current quarks as well as the Fermi vev. So in sum, (5.10) makes sense on multiple bases: it yields an empirical match to within 6 parts in 10,000; it is the product of a proton matrix with a neutron matrix; the proton matrix contains the masses and charges of two up quarks and one down quark while the neutron matrix contains the masses and charges of two down quarks and one up quark; and it is fully symmetric under both $u \leftrightarrow d$ and $p \leftrightarrow n$ interchange.

Furthermore, if we divide (5.8) by 2, we see that:

$$
\begin{aligned}
& K_{A B}(P) K_{B A}(N) / 2 \\
& =\frac{3}{2} \sqrt[4]{\frac{2}{9} v_{F}^{2} m_{u} m_{d}}=928.7853174 \mathrm{MeV}
\end{aligned}
$$

This actually falls between $m_{P}=928.9179915 \mathrm{MeV}$ and $m_{N}=927.5286457 \mathrm{MeV}$ from (5.1) and (5.2), so (5.10) clearly appears to be a correct expression for the leading terms in the neutron and proton masses. Based on this close concurrence and "threading the needle" between the neutron and proton masses with (5.11) and all of the appropriate symmetries noted in the previous paragraph, we now regard (5.10) as a meaningful (rather than coincidental) close expression for $M_{P}+M_{N}$ to $0.06 \%$.

It will simplify and clarify the calculations from here to use an uppercase $M$ notation to define what we shall hereafter refer to as "vacuum-amplified" up and down quark masses according to:

$$
\begin{gathered}
M_{u} \equiv \sqrt{\frac{2}{3} v_{F} m_{u}}=604.1751345 \mathrm{MeV}, \\
M_{d} \equiv \sqrt{\frac{1}{3} v_{F} m_{d}}=634.5784463 \mathrm{MeV} .
\end{gathered}
$$

Consequently:

$$
\sqrt{M_{u} M_{d}}=\sqrt[4]{\frac{2}{9} v_{F}^{2} m_{u} m_{d}}=619.1902116 \mathrm{MeV} .
$$

With these definitions, the neutron plus proton mass sum (5.10) may be rewritten more transparently as:

$$
\begin{aligned}
M_{N}+M_{P} & =m_{N}+m_{P}+3 m_{u}+3 m_{d} \\
& \cong 3\left(\sqrt{M_{u} M_{d}}+m_{u}+m_{d}\right)
\end{aligned}
$$

while the Koide mass matrices (5.6) and (5.7) for the neutron and proton become:

$$
\begin{gathered}
K_{A B}(P)=\left(\begin{array}{ccc}
i^{0.5} \sqrt{M_{d}} & 0 & 0 \\
0 & \sqrt{M_{u}} & 0 \\
0 & 0 & \sqrt{M_{u}}
\end{array}\right), \\
K_{A B}(N)=\left(\begin{array}{ccc}
\sqrt{M_{u}} & 0 & 0 \\
0 & i^{0.5} \sqrt{M_{d}} & 0 \\
0 & 0 & i^{0.5} \sqrt{M_{d}}
\end{array}\right) .
\end{gathered}
$$

These matrices now restore the $i^{0.5}=\frac{1}{\sqrt{2}}(1+i)$ coefficient that we excised to calculate (5.8). Thus, as in (5.8), but including this complex factor, we now take:

$$
\begin{aligned}
& K_{A B}(P) K_{B A}(N) \\
= & \operatorname{Tr}\left[\begin{array}{ccc}
i^{0.5} \sqrt{M_{d}} & 0 & 0 \\
0 & \sqrt{M_{u}} & 0 \\
0 & 0 & \sqrt{M_{u}}
\end{array}\right) \\
\times & \left.\left(\begin{array}{ccc}
\sqrt{M_{u}} & 0 & 0 \\
0 & i^{0.5} \sqrt{M_{d}} & 0 \\
0 & 0 & i^{0.5} \sqrt{M_{d}}
\end{array}\right)\right] \\
= & 3 i^{0.5} \sqrt{M_{u} M_{d}}=\frac{1}{\sqrt{2}}(1+i) 1857.570635 \mathrm{MeV}
\end{aligned}
$$

Having found a very close magnitude, we could make use of a $\sqrt{2}$ factor and continue to match the empirical data by writing $\sqrt{2} \operatorname{Re}\left(K_{A B}(P) K_{B A}(N)\right) \cong m_{P}+m_{N}$. But this just sidesteps understanding the meaning of this complex coefficient and it does not help us past the $0.06 \%$ difference that still remains between the predicted and the empirical data.

We now need to find a more fundamental way to understand this complex factor, as well as how to close the remaining $0.06 \%$ gap between the predicted and the observed neutron plus proton mass sum. That will be the subject of the next two Sections.

\section{Exact Characterization of the Neutron and Proton Masses via a Mixing Angle $\theta$ and Phase Angle $\delta$}

The complex factor $i^{0.5}=\frac{1}{\sqrt{2}}(1+i)$ which arises from the oppositely-signed up and down quark charges, as we shall now see, is actually like the subtle clue in a good detective story which, when pulled like a small thread and pursued to its logical end, eventually cracks the entire mystery. So, let us start to pull on this thread and see where it leads us. 
We first represent this factor $i^{0.5}=\frac{1}{\sqrt{2}}(1+i)$ in terms of a phase angle $\delta^{\prime}$ defined such that $\delta^{\prime} \equiv \pi / 4$, so that:

$$
i^{0.5}=\frac{1}{\sqrt{2}}(1+i)=\exp \left(i \delta^{\prime}\right)=\cos \delta^{\prime}+i \sin \delta^{\prime} .
$$

Then, we briefly rename $K \rightarrow K^{\prime}$ and use this phase to rewrite (5.18) as:

$$
\begin{aligned}
& K_{A B}^{\prime}(P) K_{B A}^{\prime}(N) \\
& =\operatorname{Tr}\left[\left(\begin{array}{ccc}
\mathrm{e}^{i \delta^{\prime}} \sqrt{M_{d}} & 0 & 0 \\
0 & \sqrt{M_{u}} & 0 \\
0 & 0 & \sqrt{M_{u}}
\end{array}\right)\right. \\
& \times\left(\begin{array}{ccc}
\sqrt{M_{u}} & 0 & 0 \\
0 & \mathrm{e}^{i \delta^{\prime}} \sqrt{M_{d}} & 0 \\
0 & 0 & \mathrm{e}^{i \delta^{\prime}} \sqrt{M_{d}}
\end{array}\right] \\
& =3 \exp \left(i \delta^{\prime}\right) \sqrt{M_{u} M_{d}}=m_{N}^{\prime}+m_{P}^{\prime}
\end{aligned}
$$

with $i^{0.5} \rightarrow \exp \left(i \delta^{\prime}\right)$ in separate matrices (5.16), (5.17) also. Then we use this to rewrite mass sum (5.15) with $i^{0.5} \rightarrow \exp \left(i \delta^{\prime}\right)$ restored as:

$$
\begin{aligned}
M_{N}^{\prime}+M_{P}^{\prime} & =m_{N}^{\prime}+m_{P}^{\prime}+3 m_{u}+3 m_{d} \\
& \cong 3\left(\sqrt{M_{u} M_{d}} \exp \left(i \delta^{\prime}\right)+m_{u}+m_{d}\right)
\end{aligned}
$$

where we have also briefly renamed $M \rightarrow M^{\prime}$ and $m_{P, N} \rightarrow m_{P, N}^{\prime}$, all with $\delta^{\prime}=\pi / 4$.

Now, (6.3) is important, because it gives us an opportunity to define a new Koide matrix $\mathrm{E}_{A B}$ which we shall refer to as the "electron generation matrix" $E$ as such:

$$
\mathrm{E}_{A B} \equiv \sqrt{3}\left(\begin{array}{ccc}
\sqrt[4]{M_{u} M_{d}} & 0 & 0 \\
0 & \sqrt{m_{u}} & 0 \\
0 & 0 & \sqrt{m_{d}}
\end{array}\right) .
$$

Then, making note of the phase $\exp \left(i \delta^{\prime}\right)$ which multiplies $\sqrt{M_{u} M_{d}}$ in (6.3) and keeping in mind how the Kobayashi and Maskawa mixing matrices are formed for three generations, we introduce a new angle $\theta_{1}$ such that $\theta_{1}^{\prime}=0$ and form a unitary matrix $U_{1}^{\prime}$ with $\mathrm{e}^{i \delta^{\prime}}$ :

$$
U_{1 A B}^{\prime} \equiv\left(\begin{array}{ccc}
\exp \left(i \delta^{\prime}\right) & 0 & 0 \\
0 & \cos \theta_{1}^{\prime} & \sin \theta_{1}^{\prime} \\
0 & -\sin \theta_{1}^{\prime} & \cos \theta_{1}^{\prime}
\end{array}\right)=\left(\begin{array}{ccc}
i^{0.5} & 0 & 0 \\
0 & 1 & 0 \\
0 & 0 & 1
\end{array}\right) .
$$

So (6.5) sandwich-multiplied by (6.4) simply generalizes the appearance of the term $i^{0.5} \sqrt{M_{u} M_{d}}$ in (5.18). But now let us permit both $\delta$ and $\theta$ to rotate freely, $\theta^{\prime} \rightarrow \theta, \quad \delta^{\prime} \rightarrow \delta$. Then, using (6.4) and (6.5), we may form the neutron plus proton mass sun according to Equation (6.6) at the bottom of the page.

For the special case where $\theta \rightarrow \theta^{\prime}=0$ and $\delta \rightarrow \delta^{\prime}=\pi / 4$, (6.6) precisely reproduces (6.3). But in (6.6) we have removed the approximation sign $\cong$ that was in (6.3), because we are now going to define the angles $\theta, \delta$ so as to precisely match up with the empirical values of the neutron and proton masses. That is, just as (1.4) is an exact formula for the proton-neutron mass difference, we shall now regard (6.6) as an exact formula for the neutron plus proton mass sum, with the numerical values of $\theta, \delta$ defined by empirical data so as to make this an exact fit.

Now before we proceed, let us pause to make clear, the cascading detective work we have just done: We have used the matrix $\operatorname{diag} U=\left(i^{0.5}, 1,1\right)$ implicit in $(6.3)$ and explicit in (6.5) as a hint that there exists a matrix $\operatorname{diag} U=\left(\exp \left(i \delta^{\prime}\right), 1,1\right)$ with $\delta^{\prime}=\pi / 4$. Then we use $\operatorname{diag} U=\left(\exp \left(i \delta^{\prime}\right), 1,1\right)$ as a further hint that there exists a matrix (6.5). Then we allow both of these angles to freely rotate to form (6.6) which generalizes (6.3). Following all of this, we will use these freely rotated angles to permit the otherwise close relationship (6.3) to be fitted exactly by empirically choosing these angles so as to yield an exact fit.

But before we do this, however, there is a final, deep cascade to this hint, which is to recognize that (6.5) with angles free to rotate is one of the three matrices used to define the CKM matrices used for electroweak generation mixing, see (7.11) in [8], and in particular, is the matrix that is use to introduce the phase angle responseble for $C P$ violation. We also see that (6.4) is strictly a function of the first (electron generation) quark masses and the Fermi vev which makes its upper left component $\sqrt[4]{M_{u} M_{d}}$ containing the "vacuum-enhanced" quark

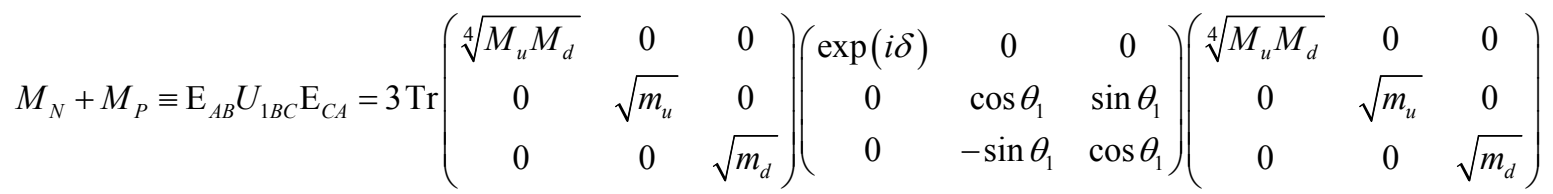

$$
\begin{aligned}
& =3 \operatorname{Tr}\left(\begin{array}{ccc}
\sqrt{M_{u} M_{d}} \exp (i \delta) & 0 & 0 \\
0 & m_{u} \cos \theta_{1} & \sqrt{m_{u} m_{d}} \sin \theta_{1} \\
0 & -\sqrt{m_{u} m_{d}} \sin \theta_{1} & m_{d} \cos \theta_{1}
\end{array}\right)=3\left(\sqrt{M_{u} M_{d}} \exp (i \delta)+m_{u} \cos \theta_{1}+m_{d} \cos \theta_{1}\right)
\end{aligned}
$$


masses substantially larger than its middle and lower right components $\sqrt{m_{u}}$ and $\sqrt{m_{d}}$.

Because CKM mixing has two more matrices and also mixes two more generations, let us now form two more matrices $\mathrm{M}$ and $\mathrm{T}$ analogous to (6.4) for the muon and tauon generation of quarks, following the pattern for mixing in the original parameterization of Kobayashi and Maskawa. Thus, we put the large components $\sqrt[4]{M_{c} M_{s}}$ and $\sqrt[4]{M_{t} M_{b}}$ into the lower right positions. And, as a matter of convention, we keep the up (electric charge $=$ $+2 / 3$ ) series of mass terms in the middle position. Thus we define the muon and tauon generation matrices as:

$$
\begin{gathered}
\mathrm{M}_{A B} \equiv \sqrt{3}\left(\begin{array}{ccc}
\sqrt{m_{s}} & 0 & 0 \\
0 & \sqrt{m_{c}} & 0 \\
0 & 0 & \sqrt[4]{M_{c} M_{s}}
\end{array}\right) ; \\
\mathrm{T}_{A B} \equiv \sqrt{3}\left(\begin{array}{ccc}
\sqrt{m_{b}} & 0 & 0 \\
0 & \sqrt{m_{t}} & 0 \\
0 & 0 & \sqrt[4]{M_{t} M_{b}}
\end{array}\right) .
\end{gathered}
$$

At the same time, analogously to (5.12) and (5.13), we define the vacuum-enhanced higher-generation quark masses:

$$
\begin{aligned}
& M_{c} \equiv \sqrt{\frac{2}{3} v m_{c}}=14,467 \mathrm{MeV} \text {, } \\
& M_{s} \equiv \sqrt{\frac{1}{3} v m_{s}}=2792 \mathrm{MeV} \text {, } \\
& \mathrm{T}_{A B} U_{3 B C} \mathrm{~T}_{C A}=3 \operatorname{Tr}\left(\begin{array}{cc}
m_{b} \cos \theta_{3} & \sqrt{m_{b} m_{t}} \sin \theta_{3} \\
-\sqrt{m_{b} m_{t}} \sin \theta_{3} & m_{t} \cos \theta_{3} \\
0 & 0
\end{array}\right.
\end{aligned}
$$$$
\mathrm{M}_{A B} U_{2 B C} \mathrm{M}_{C A}=3 \operatorname{Tr}\left(\begin{array}{ccc}
m_{s} \cos \theta_{2} & \sqrt{m_{s} m_{c}} \sin \theta_{2} & 0 \\
-\sqrt{m_{s} m_{c}} \sin \theta_{2} & m_{c} \cos \theta_{2} & 0 \\
0 & 0 & \sqrt{M_{c} M_{s}}
\end{array}\right)=3\left(\sqrt{M_{c} M_{s}}+m_{c} \cos \theta_{2}+m_{s} \cos \theta_{2}\right),
$$

Then, we multiply all three of (6.6), (6.15) and (6.16) together in the same manner that the Cabibbo mixing matrices are formed, again see (7.11) in [8], to obtain a

$$
\begin{gathered}
M_{t} \equiv \sqrt{\frac{2}{3} v m_{t}}=168,758 \mathrm{MeV}, \\
M_{b} \equiv \sqrt{\frac{1}{3} v m_{b}}=18,522 \mathrm{MeV},
\end{gathered}
$$

which yields the higher-generation analogues to (5.14):

$$
\begin{aligned}
& \sqrt{M_{c} M_{s}}=6356 \mathrm{MeV}, \\
& \sqrt{M_{t} M_{b}}=55,908 \mathrm{MeV} .
\end{aligned}
$$

These values are calculated from the PDG data [10] laid out prior to (3.12), rounded to the nearest $\mathrm{MeV}$ (recognizing substantial experimental uncertainties).

We also define two more matrices analogous to (6.5) for the second and third generations in same manner as is used to form the CKM mixing matrices, again see (7.11) in [8]:

$$
\begin{aligned}
& U_{2 A B} \equiv\left(\begin{array}{ccc}
\cos \theta_{2} & \sin \theta_{2} & 0 \\
-\sin \theta_{2} & \cos \theta_{2} & 0 \\
0 & 0 & 1
\end{array}\right) ; \\
& U_{3 A B} \equiv\left(\begin{array}{ccc}
\cos \theta_{3} & \sin \theta_{3} & 0 \\
-\sin \theta_{3} & \cos \theta_{3} & 0 \\
0 & 0 & 1
\end{array}\right) .
\end{aligned}
$$

Then, analogously to (6.6), for the second and third generations, respectively, we form:

$$
\begin{aligned}
& \Theta \equiv \mathrm{M} \cdot U_{2} \cdot \mathrm{M} \cdot \mathrm{E} \cdot U_{1} \cdot \mathrm{E} \cdot \mathrm{T} \cdot U_{3} \cdot \mathrm{T} \\
& =27\left(\begin{array}{lll}
-m_{u} \sqrt{m_{s} m_{c}} \sqrt{m_{b} m_{t}} c_{1} s_{2} s_{3} & m_{u} \sqrt{m_{s} m_{c}} m_{t} c_{1} s_{2} c_{3} & \\
+\sqrt{M_{u} M_{d}} m_{s} m_{b} c_{2} c_{3} \mathrm{e}^{i \delta} & +\sqrt{M_{u} M_{d}} m_{s} \sqrt{m_{b} m_{t}} c_{2} s_{3} \mathrm{e}^{i \delta} & \sqrt{m_{u} m_{d}} \sqrt{m_{s} m_{c}} \sqrt{M_{t} M_{b}} s_{1} s_{2} \\
-m_{u} m_{c} \sqrt{m_{b} m_{t}} c_{1} c_{2} s_{3} & m_{u} m_{c} m_{t} c_{1} c_{2} c_{3} & \\
-\sqrt{M_{u} M_{d}} \sqrt{m_{s} m_{c}} m_{b} s_{2} c_{3} \mathrm{e}^{i \delta} & -\sqrt{M_{u} M_{d}} \sqrt{m_{s} m_{c}} \sqrt{m_{b} m_{t}} s_{2} s_{3} \mathrm{e}^{i \delta} & \sqrt{m_{u} m_{d}} m_{c} \sqrt{M_{t} M_{b}} s_{1} c_{2} \\
\sqrt{m_{u} m_{d}} \sqrt{M_{c} M_{s}} \sqrt{m_{b} m_{t} s_{1} s_{3}} & -\sqrt{m_{u} m_{d}} \sqrt{M_{c} M_{s} m_{t} s_{1} c_{3}} & m_{d} \sqrt{M_{c} M_{s}} \sqrt{M_{t} M_{b}} c_{1}
\end{array}\right)
\end{aligned}
$$


This master matrix contains all six of the quark masses in all three generations, all three of the real mixing angles and the one phase angle that appears when the three generations are mixed, and implied in the vacuum-enhanced mass terms, the Fermi vev and the electric charges of all of these quarks. If all of the masses are set to equal 1, this reduces to the usual generational mixing matrix in the original parameterization of Kobayashi and Maskawa, seen in, e.g., (7.11) in [8]. In the circumstance where $s_{2}=0, s_{3}=0$, this reduces to:

$$
\Theta=27\left(\begin{array}{ccc}
\sqrt{M_{u} M_{d}} m_{s} m_{b} \mathrm{e}^{i \delta} & 0 & 0 \\
0 & m_{u} m_{c} m_{t} \cos \theta_{1} & \sqrt{m_{u} m_{d}} m_{c} \sqrt{M_{t} M_{b}} \sin \theta_{1} \\
0 & -\sqrt{m_{u} m_{d}} \sqrt{M_{c} M_{s}} m_{t} \sin \theta_{1} & m_{d} \sqrt{M_{c} M_{s}} \sqrt{M_{t} M_{b}} \cos \theta_{1}
\end{array}\right) .
$$

and in the further circumstance where all of the second and third generation masses are set to 1 , this further reduces to 9 times the matrix shown in (6.6):

$$
\Theta=27\left(\begin{array}{ccc}
\sqrt{M_{u} M_{d}} \mathrm{e}^{i \delta} & 0 & 0 \\
0 & m_{u} \cos \theta_{1} & \sqrt{m_{u} m_{d}} \sin \theta_{1} \\
0 & -\sqrt{m_{u} m_{d}} \sin \theta_{1} & m_{d} \cos \theta_{1}
\end{array}\right) .
$$

So in this particular special case, (6.17) even contains the neutron plus proton mass sum of (6.6):

$$
\frac{1}{9} \operatorname{Tr} \Theta=3\left(\sqrt{M_{u} M_{d}} \exp (i \delta)+m_{u} \cos \theta_{1}+m_{d} \cos \theta_{1}\right)=M_{N}+M_{P} !
$$

So this neutron plus proton mass sum now is a special case of (6.17) which includes all the generation mixing angles and all the quark masses and their electric charges and the Fermi vev!

Consequently, one expects that (6.17) can be used to gain substantial new insights into fermion and baryon masses generally. And all of this emerges in cascade fashion from the simple hint of a matrix with $\operatorname{diag} U=\left(i^{0.5}, 1,1\right)$ in the neutron plus proton mass for- mula (6.3), with the $i^{0.5}$ itself having emerged from the simple fact that up and down quarks have oppositelysigned charges which led to terms containing $\sqrt[4]{-1}$ when we formed Koide matrices to represent masses. Such is the nature of this detective mystery!

With the important contextual digression of (6.7) through (6.20) as backdrop, we now return to solve (1.4) and (6.6) as simultaneous equations, that is, we now solve the simultaneous equation set:

$$
\left\{\begin{array}{l}
M_{P}+M_{N}=3\left(\sqrt{M_{u} M_{d}} \exp (i \delta)+m_{u} \cos \theta_{1}+m_{d} \cos \theta_{1}\right) \\
M_{N}-M_{P}=m_{u}-\left(3 m_{d}+2 \sqrt{m_{\mu} m_{d}}-3 m_{u}\right) /(2 \pi)^{\frac{3}{2}}
\end{array}\right.
$$

We now need no more than elementary algebra to determine that the neutron and proton masses, separately, are each given by:

$$
\begin{aligned}
& M_{N}=\frac{1}{2}\left(3\left(\sqrt{M_{u} M_{d}} \exp (i \delta)+\cos \theta_{1}\left(m_{u}+m_{d}\right)\right)+m_{u}-\left(3 m_{d}+2 \sqrt{m_{\mu} m_{d}}-3 m_{u}\right) /(2 \pi)^{\frac{3}{2}}\right) \\
& M_{P}=\frac{1}{2}\left(3\left(\sqrt{M_{u} M_{d}} \exp (i \delta)+\cos \theta_{1}\left(m_{u}+m_{d}\right)\right)-m_{u}+\left(3 m_{d}+2 \sqrt{m_{\mu} m_{d}}-3 m_{u}\right) /(2 \pi)^{\frac{3}{2}}\right)
\end{aligned}
$$

These can be made into exact theoretical expressions for the neutron and proton mass by solving for $\theta_{1}, \delta$, to find their empirical values based on the empirical neutron and proton masses. Let's now do so.
Because each of (6.22) contains a complex phase, we will need to form the square modulus magnitude $|M|^{2}=M^{*} M$ of these masses. So first we deduce: 


$$
\begin{aligned}
4\left|M_{N}\right|^{2}= & 9 M_{u} M_{d}+6 \cos \delta \sqrt{M_{u} M_{d}}\left(3 \cos \theta_{1}\left(m_{u}+m_{d}\right)+m_{u}-\left(3 m_{d}+2 \sqrt{m_{\mu} m_{d}}-3 m_{u}\right) /(2 \pi)^{\frac{3}{2}}\right) \\
& +\left(3 \cos \theta_{1}\left(m_{u}+m_{d}\right)+m_{u}-\left(3 m_{d}+2 \sqrt{m_{\mu} m_{d}}-3 m_{u}\right) /(2 \pi)^{\frac{3}{2}}\right)^{2} ; \\
4\left|M_{P}\right|^{2}= & 9 M_{u} M_{d}+6 \cos \delta \sqrt{M_{u} M_{d}}\left(3 \cos \theta_{1}\left(m_{u}+m_{d}\right)-m_{u}+\left(3 m_{d}+2 \sqrt{m_{\mu} m_{d}}-3 m_{u}\right) /(2 \pi)^{\frac{3}{2}}\right) \\
& +\left(3 \cos \theta_{1}\left(m_{u}+m_{d}\right)-m_{u}+\left(3 m_{d}+2 \sqrt{m_{\mu} m_{d}}-3 m_{u}\right) /(2 \pi)^{\frac{3}{2}}\right)^{2}
\end{aligned}
$$

Now we solve these as simultaneous equations for $\theta_{1}$ and $\delta$. First we restructure (6.23) in terms of $\delta$ to arrive at:

$$
\begin{gathered}
\cos \delta=\frac{4\left|M_{N}\right|^{2}-9 M_{u} M_{d}-\left(3 \cos \theta_{1}\left(m_{u}+m_{d}\right)+m_{u}-\left(3 m_{d}+2 \sqrt{m_{\mu} m_{d}}-3 m_{u}\right) /(2 \pi)^{\frac{3}{2}}\right)^{2}}{6 \sqrt{M_{u} M_{d}}\left(3 \cos \theta_{1}\left(m_{u}+m_{d}\right)+m_{u}-\left(3 m_{d}+2 \sqrt{m_{\mu} m_{d}}-3 m_{u}\right) /(2 \pi)^{\frac{3}{2}}\right)} \\
\cos \delta=\frac{4\left|M_{P}\right|^{2}-9 M_{u} M_{d}-\left(3 \cos \theta_{1}\left(m_{u}+m_{d}\right)-m_{u}+\left(3 m_{d}+2 \sqrt{m_{\mu} m_{d}}-3 m_{u}\right) /(2 \pi)^{\frac{3}{2}}\right)^{2}}{6 \sqrt{M_{u} M_{d}}\left(3 \cos \theta_{1}\left(m_{u}+m_{d}\right)-m_{u}+\left(3 m_{d}+2 \sqrt{m_{\mu} m_{d}}-3 m_{u}\right) /(2 \pi)^{\frac{3}{2}}\right)}
\end{gathered}
$$

We now set these two $\cos \delta$ equal to one another to eliminate $\delta$ and solve for $\theta$. It will be easier to see the underlying structure of these equations as well as solve them if we write (6.24) above as:

$$
\begin{aligned}
C \cos \delta & =\frac{N-\left(B \cos \theta_{1}+A\right)^{2}}{\left(B \cos \theta_{1}+A\right)} \\
& =\frac{P-\left(B \cos \theta_{1}-A\right)^{2}}{\left(B \cos \theta_{1}-A\right)}
\end{aligned}
$$

using the following substitution of variables:

$$
\begin{aligned}
& N \equiv 4\left|M_{N}\right|^{2}-9 M_{u} M_{d} ; \\
& P \equiv 4\left|M_{P}\right|^{2}-9 M_{u} M_{d} \\
& A \equiv m_{u}-\left(3 m_{d}+2 \sqrt{m_{\mu} m_{d}}-3 m_{u}\right) /(2 \pi)^{\frac{3}{2}} ; \\
& B \equiv 3\left(m_{u}+m_{d}\right) ; C \equiv 6 \sqrt{M_{u} M_{d}}
\end{aligned}
$$

Next, we reduce the second and third terms of (6.25) successively in five steps as follows:

$$
\begin{aligned}
& \text { 1) }:\left(N-\left(B \cos \theta_{1}+A\right)^{2}\right)\left(B \cos \theta_{1}-A\right)=\left(P-\left(B \cos \theta_{1}-A\right)^{2}\right)\left(B \cos \theta_{1}+A\right) \\
& \text { 2) }: N\left(B \cos \theta_{1}-A\right)-\left(B^{2} \cos ^{2} \theta_{1}-A^{2}\right)\left(B \cos \theta_{1}+A\right)=P\left(B \cos \theta_{1}+A\right)-\left(B^{2} \cos ^{2} \theta_{1}-A^{2}\right)\left(B \cos \theta_{1}-A\right) \\
& \text { 3) }: \frac{N\left(B \cos \theta_{1}-A\right)}{\left(B^{2} \cos ^{2} \theta_{1}-A^{2}\right)}-A=\frac{P\left(B \cos \theta_{1}+A\right)}{\left(B^{2} \cos ^{2} \theta_{1}-A^{2}\right)}+A \\
& \text { 4) }: N\left(B \cos \theta_{1}-A\right)-A\left(B^{2} \cos ^{2} \theta_{1}-A^{2}\right)=P\left(B \cos \theta_{1}+A\right)+A\left(B^{2} \cos ^{2} \theta_{1}-A^{2}\right) \\
& \text { 5) }: 0=\left\{2 A B^{2}\right\} \cos ^{2} \theta_{1}-\{B(N-P)\} \cos \theta_{1}+\left\{A(N+P)-2 A^{3}\right\}
\end{aligned}
$$

In the final step, we arrive at a quadratic for $\cos \theta_{1}$, and so obtain a solution via the quadratic equation. Then, we use the variables (6.26) including the empirical masses of the neutron and proton, to calculate that:

$$
\begin{aligned}
\cos \theta_{1} & =\frac{N-P-\sqrt{(N-P)^{2}-8\left(A^{2}(N+P)-2 A^{4}\right)}}{4 A B} \\
& =0.9474541242
\end{aligned}
$$

Additionally, $\sin \theta_{1}=0.31989167$. In the above, we use the negative root, because this yields $-1 \leq \cos \theta_{1} \leq 1$. This means the empirically-determined value of $\theta_{1}$ is:

$$
\begin{aligned}
\theta_{1} & =0.32561515 \mathrm{rad}=18.65637386^{\circ} \\
& =\pi / 9.64817715
\end{aligned}
$$

We shall refer to $\cos \theta_{1}=0.9474541242$ in (6.28) used to precisely fit (6.22) to the observed neutron and 
proton masses as the "nucleon fitting angle". In the next Section we shall show how to tie this angle to the observed CKM mixing angles, so it is not a "new" angle, but is related to other known mixing data.

Now, we use (6.28) in (6.25) to solve for $\delta$, and calculate to find that:

$$
\begin{aligned}
\cos \delta= & C\left(\left(N-P-\sqrt{(N-P)^{2}-8\left(A^{2}(N+P)-2 A^{4}\right)}\right) / 4 A+A\right) \\
= & \frac{P-\left(\left(N-P-\sqrt{(N-P)^{2}-8\left(A^{2}(N+P)-2 A^{4}\right)}\right) / 4 A-A\right)^{2}}{C\left(\left(N-P-\sqrt{(N-P)^{2}-8\left(A^{2}(N+P)-2 A^{4}\right)}\right) / 4 A-A\right)}=1
\end{aligned}
$$

This numerical calculation reveals that $\cos \delta=1$, exactly, to all decimal places, so the phase factor $\delta=0$. This means that when the variables in (6.26) are substituted into (6.30), the extremely unwieldy-looking resulting expression will reduce to 1 identically! So to the extent that $\delta$ may be a CP-violating phase, and given that $\delta=0$ is a deduced result for the neutron and proton masses (6.22), this deductively tells us that there are no CP-violating effects associated with neutron and proton.
This is validated by empirical data which shows the mass of the antiproton is equal to that of the proton, and the mass of the antineutron is equal to that of the neutron, see, e.g., $[12,13]$. So, we take (6.22) to be exact formulations of the neutron and proton masses, in the circumstance where empirically-determined angle $\cos \theta_{1}=0.9474541242$ and CP-violating phase $\delta=0$.

So we now return to (6.22), set $\delta=0$, and so obtain our final expressions for the neutron and proton masses:

$$
\begin{aligned}
& M_{N}=\frac{1}{2}\left(3\left(\sqrt{M_{u} M_{d}}+\cos \theta_{1}\left(m_{u}+m_{d}\right)\right)+m_{u}-\left(3 m_{d}+2 \sqrt{m_{\mu} m_{d}}-3 m_{u}\right) /(2 \pi)^{\frac{3}{2}}\right) \\
& M_{P}=\frac{1}{2}\left(3\left(\sqrt{M_{u} M_{d}}+\cos \theta_{1}\left(m_{u}+m_{d}\right)\right)-m_{u}+\left(3 m_{d}+2 \sqrt{m_{\mu} m_{d}}-3 m_{u}\right) /(2 \pi)^{\frac{3}{2}}\right)
\end{aligned}
$$

which are exact relations with the empirical substitution $\cos \theta_{1}=0.9474541242$.

These relationships (6.31), in turn, now enable us to go back to the masses (nuclear weights) for the 1s nuclides predicted in [5] to high accuracy and rewrite (8.6), (8.1), (8.3) and (8.5) of [5], respectively, as:

$$
\begin{aligned}
{ }_{1}^{2} M & =M_{P}+M_{N}-m_{u}=3\left(\sqrt{M_{u} M_{d}}+\cos \theta_{1}\left(m_{u}+m_{d}\right)\right)-m_{u}, \\
{ }_{1}^{3} M & =M_{P}+2 M_{N}-4 m_{u}+2 \sqrt{m_{\mu} m_{d}} /(2 \pi)^{\frac{3}{2}} \\
& =\frac{1}{2}\left(9\left(\sqrt{M_{u} M_{d}}+\cos \theta_{1}\left(m_{u}+m_{d}\right)\right)-7 m_{u}-\left(3 m_{d}-2 \sqrt{m_{\mu} m_{d}}-3 m_{u}\right)(2 \pi)^{\frac{3}{2}}\right) \\
{ }_{2}^{3} M & =2 M_{P}+M_{N}-2 m_{u}-\sqrt{m_{u} m_{d}} \\
& =\frac{1}{2}\left(9\left(\sqrt{M_{u} M_{d}}+\cos \theta_{1}\left(m_{u}+m_{d}\right)\right)-5 m_{u}-2 \sqrt{m_{u} m_{d}}+\left(3 m_{d}+2 \sqrt{m_{\mu} m_{d}}-3 m_{u}\right) /(2 \pi)^{\frac{3}{2}}\right) \\
{ }_{2}^{4} M & =2 M_{P}+2 M_{N}-6 m_{u}-6 m_{d}+\left(10 m_{d}+10 m_{u}+16 \sqrt{m_{u} m_{d}}\right) /(2 \pi)^{\frac{3}{2}}+2 \sqrt{m_{u} m_{d}} \\
& =6\left(\sqrt{M_{u} M_{d}}+\cos \theta_{1}\left(m_{u}+m_{d}\right)-m_{u}-m_{d}\right)+2 \sqrt{m_{u} m_{d}}+\left(10 m_{d}+10 m_{u}+16 \sqrt{m_{u} m_{d}}\right) /(2 \pi)^{\frac{3}{2}}
\end{aligned}
$$

Now, ${ }_{Z}^{A} B_{0}=Z M_{P}+N M_{N}-{ }_{Z}^{A} M$, which is binding energy $B_{0}$ for any given nuclide with $Z$ protons and $N$ neutrons hence $A=Z+N$ nucleons, thus $N-Z=A-2 Z$, may also be rewritten generally in relation to nuclear 
weights using (6.31), in the form:

$$
{ }_{Z}^{A} B_{0}+{ }_{Z}^{A} M=\frac{1}{2}\left(3 A\left(\sqrt{M_{u} M_{d}}+\cos \theta_{1}\left(m_{u}+m_{d}\right)\right)+(A-2 Z)\left(m_{u}-\left(\frac{3 m_{d}+2 \sqrt{m_{\mu} m_{d}}-3 m_{u}}{(2 \pi)^{\frac{3}{2}}}\right)\right)\right)
$$

One final exploratory exercise of interest is to return to the master mass and mixing matrix $\Theta$ in (6.17) and set $\theta_{2}=\theta_{3}=\delta=0$ while using $\cos \theta_{1}=0.9474541242$

found in (6.28). In this circumstance, (6.17) reduces to:

$$
\Theta=27\left(\begin{array}{ccc}
\sqrt{M_{u} M_{d}} m_{s} m_{b} & 0 & 0 \\
0 & m_{u} m_{c} m_{t} \cos \theta_{1} & 0 \\
0 & 0 & m_{d} \sqrt{M_{c} M_{s}} \sqrt{M_{t} M_{b}} \cos \theta_{1}
\end{array}\right) .
$$

This is in dimensions of mass ${ }^{3}$. If we take the cubed root, and divide by 2 (because we know that this originated with the neutron plus proton mass sum) to get mass numbers that should be related to individual baryons, we find

$$
\frac{1}{2} \operatorname{diag} \sqrt[3]{\Theta}=(939.72 \mathrm{MeV}, 1163 \mathrm{MeV}, 1773 \mathrm{MeV})
$$

(and we also get a coefficient $\sqrt[3]{27} / 2=3 / 2$, back to Koide!). This first entry is very close to the neutron mass 939.565379 MeV which would not be expected a priori, but this is because $\sqrt{m_{s} m_{b}}=630 \mathrm{MeV}$ which is not too far from $\sqrt{M_{u} M_{d}}=619 \mathrm{MeV}$. Perhaps this is yet another close relationship among fermion masses!? The second entry at $1163 \mathrm{MeV}$, which would become smaller when $\theta_{2} \neq 0, \theta_{3} \neq 0$, is only about $4 \%$ larger than the mass of the $\Lambda_{0}(u d s)=1115.683 \mathrm{MeV}$ baryon, which could readily be compensated by non-zero $\theta_{2}, \theta_{3}$ angles as well as experimental errors in the charm and top quark masses. The final entry at $1773 \mathrm{MeV}$, is perhaps suggestive of the $\Omega_{-}(s s s)=1672.45 \mathrm{MeV}$ baryon mass, however, contra, there are no omitted angles and somewhere we should expect to come across a baryon with a third generation quark.

These (6.37) relationships are simply pointed out in an exploratory spirit, and it is to be noted that $\Theta$ in (6.17) is just one representation of a mass/mixing matrix and that one can also vary the way in which one sets up the Koide triplets (6.4) and (6.7), so as to be able to obtain this $\Theta$ matrix in several different representations. Whatever the correct fits may turn out to be with various higher-generation baryons, it should be clear that the master matrix (6.17) and like matrices that can be similarly constructed are an exceedingly useful tool for trying to develop and fit mutual relationships among mixing angles, $\mathrm{CP}$ violating phases, and quark and baryon masses.

\section{Relation of the Nucleon Fitting Angle $\theta$ to the CKM Mixing Angles}

Following the development in the last Section, the nucleon fitting angle $\cos \theta_{1}=0.9474541242$ found in (6.28) is a new empirical parameter that enables us to precisely formulate the neutron and proton masses using (6.31). While this is an important step forward in understanding the neutron and proton masses, it would be even better if this angle could be related in some way to the empirically-known CKM quark mixing angles, which could then relate the neutron and proton masses themselves to the CKM angles. This is highly preferable to having $\cos \theta_{1}$ be a new, separate parameter.

Toward this end, we first write the CKM matrix with the "standard choice" of angles and its empirical values from PDG's [14] as:

$$
\begin{aligned}
V & =\left(\begin{array}{ccc}
V_{u d} & V_{u s} & V_{u b} \\
V_{c d} & V_{c s} & V_{c b} \\
V_{t d} & V_{t s} & V_{t b}
\end{array}\right)=\left(\begin{array}{ccc}
c_{12} c_{13} & s_{12} c_{13} & s_{13} \mathrm{e}^{-i \delta} \\
-s_{12} c_{23}-c_{12} s_{23} s_{13} \mathrm{e}^{i \delta} & c_{12} c_{23}-s_{12} s_{23} s_{13} \mathrm{e}^{i \delta} & s_{23} c_{13} \\
s_{12} S_{23}-c_{12} c_{23} s_{13} \mathrm{e}^{i \delta} & -c_{12} s_{23}-s_{12} c_{23} s_{13} \mathrm{e}^{i \delta} & c_{23} c_{13}
\end{array}\right) . \\
& =\left(\begin{array}{ccc}
0.97427 \pm 0.00015 & 0.22534 \pm 0.00065 & 0.00351_{-0.00014}^{+0.00015} \\
-0.22520 \pm 0.00065 & 0.97344 \pm 0.00016 & 0.0412_{-0.0005}^{+0.0011} \\
-0.00867_{-0.00031}^{+0.00029} & -0.0404_{-0.0005}^{+0.0011} & 0.999146_{-0.000046}^{+0.000021}
\end{array}\right)
\end{aligned}
$$

(We use a negative sign for the three lower-left empirical entries to match the negative values in the terms which the standard CKM matrix takes on when the angles are between 0 and $\pi / 2$.) Now, $\cos \theta_{1}=$ 0.9474541242 does not fit any particular one of these elements. But what is of interest is the determinant 
$|V|$ which may be calculated from the CKM mixing and phase angles $\theta_{i j}$ and $\delta$ to be:

$$
\begin{aligned}
|V|= & V_{u d} V_{c s} V_{t b}+V_{u s} V_{c b} V_{t d}+V_{u b} V_{c d} V_{t s} \\
& -V_{u b} V_{c s} V_{t d}-V_{u s} V_{c d} V_{t b}-V_{u d} V_{c b} V_{t s}=1
\end{aligned}
$$

and which contains invariant expressions of interest (See also [15] which cleverly connects this determinant, when real as in the standard angle choice (7.1), to the Jarlskog determinant). Specifically, if we employ the mean experimental values in (7.1), we find that sum of the three positively-signed $(+)$ terms in the determinant, denoted $|V|_{+}$, which is an invariant containing all nine matrix elements, and which we shall refer to as the "major determinant," is determined from the empirical data in (7.1) to be:

$$
\begin{aligned}
|V|_{+} & =V_{u d} V_{c s} V_{t b}+V_{u s} V_{c b} V_{t d}+V_{u b} V_{c d} V_{t s} \\
& =0.947535
\end{aligned}
$$

This major determinant is very close to $\cos \theta_{1}=$ 0.947454 , truncated to the known precision of $|V|_{+}$. In fact we find $|V|_{+}=0.947192=\cos \theta_{1}-0.000262$ if we use the lower bounds of all the experimental error ranges in (7.1), and $|V|_{+}=0.947854=\cos \theta_{1}+0.000400$ if we use upper bounds. So this is within experimental errors. Therefore, using $\cos \theta_{1}=0.947454$ as the baseline against which to compare $|V|_{+}$, we find that:

$$
|V|_{+}=\cos \theta_{1-0.000262}^{+0.000400}=0.947454_{-0.000262}^{+0.000400} \text {. }
$$

This means that the nucleon mixing angle $\cos \theta_{1}$ is related to the invariant scalar $|V|_{+}$according to:

$$
\begin{aligned}
\cos \theta_{1} & =|V|_{+}=V_{u d} V_{c s} V_{t b} \\
& +V_{u s} V_{c b} V_{t d}+V_{u b} V_{c d} V_{t s}
\end{aligned}
$$

which is well within experimental errors! If we now take this to be a meaningful relationship given that it falls well within experimental errors, this means that we can go back to (6.31) and use (7.5) to rewrite the neutron and proton masses completely in terms of the CKM matrix elements, and specifically in terms of the major determinant $|V|_{+}$, according to:

$$
\begin{aligned}
M_{N}= & \frac{1}{2}\left(3\left(\sqrt{M_{u} M_{d}}+|V|_{+} \cdot\left(m_{u}+m_{d}\right)\right)+m_{u}\right. \\
& \left.-\left(3 m_{d}+2 \sqrt{m_{\mu} m_{d}}-3 m_{u}\right) /(2 \pi)^{\frac{3}{2}}\right) \\
M_{P}= & \frac{1}{2}\left(3\left(\sqrt{M_{u} M_{d}}+|V|_{+} \cdot\left(m_{u}+m_{d}\right)\right)-m_{u}\right. \\
& \left.+\left(3 m_{d}+2 \sqrt{m_{\mu} m_{d}}-3 m_{u}\right) /(2 \pi)^{\frac{3}{2}}\right)
\end{aligned}
$$

This now connects the proton and neutron masses to the major determinant $|V|_{+}$which is an invariant of the
CKM mixing matrix $V$. This not only closes the $0.06 \%$ difference of (5.18) between the predicted and the empirical neutron and proton masses using $\cos \theta_{1}$, but it connects $\cos \theta_{1}$ to the CKM mixing angles so that (7.6) now specifies the exact masses of the free neutron and proton as a function of the up and down masses and charges and the Fermi vev and the CKM quark mixing angles without introducing any new physical parameters to do so! Because $\cos \theta_{1}=0.9474541242$ is known with better precision than $|V|_{+}=0.947535$, we then use $\cos \theta_{1}$ as the basis for specifying $|V|_{+}$, i.e., we now set:

$$
|V|_{+} \equiv \cos \theta_{1}=0.9474541242 \text {, }
$$

which is then a further ingredient used to tighten the empirical data in (7.1).

Further, because $|V|_{+}$injects into the proton and neutron masses an imaginary term with a Jarlskog determinant $J=c_{13}^{2} c_{12} c_{23} s_{12} s_{13} s_{23} \sin \delta_{C K M}$ (which may be calculated using the angles in (7.1) with $\delta \rightarrow \delta_{C K M}$ ), and if we wish to maintain the proton and neutron masses to be entirely real based on $\cos \delta=1$ (the "nucleon phase angle" $\left.\delta \neq \delta_{C K M}\right)$ deduced in (6.30), then we can achieve this by restoring the phase to the vacuum-enhanced mass term as in (6.21), i.e., by restoring $\sqrt{M_{u} M_{d}} \rightarrow \sqrt{M_{u} M_{d}} \exp (i \delta)$, and then choosing $\delta$ in $i \sqrt{M_{u} M_{d}} \sin \delta$ to absorb the terms with the Jarlskog determinant, again see [15] which shows how the Jarlskog determinant is "the imaginary part of any one element among the six components of determinant of $V$.. when the whole determinant is made real" as it is in (7.2). Specifically, referring to (7.6), this means that one would set $i \sin \delta \cdot \sqrt{M_{u} M_{d}}+\operatorname{Im}|V|_{+} \cdot\left(m_{u}+m_{d}\right)=0$ to maintain $\mathrm{CP}$ symmetry for the neutron and proton. Given that $\operatorname{Im}|V|_{+}=-3 J$, this means that:

$$
\begin{aligned}
\sin \delta & \equiv 3 J \frac{m_{u}+m_{d}}{\sqrt{M_{u} M_{d}}} \\
& =3 c_{13}^{2} c_{12} c_{23} s_{12} s_{13} s_{23} \sin \delta_{C K M} \frac{m_{u}+m_{d}}{\sqrt{M_{u} M_{d}}}
\end{aligned}
$$

will define a very tiny phase in the term

$\sqrt{M_{u} M_{d}} \exp (i \delta)$ in the proton and neutron masses such that these masses remain real and thus maintain CP symmetry. While beyond the scope of this paper, this could provide additional insight into the so-called "strong CP problem.

Finally, as regards fermion masses, if we write each elementary fermion mass $m_{f}$ in terms of the Fermi vev using a dimensionless coupling $G_{f}$ as $\sqrt{2} m_{f} \equiv G_{f} v_{F}$, see, e.g., (15.32) of [16], then use these relationships in (6.17) for $\Theta$ or a similarly-formed matrix in a CKM representation (such as (7.1)), we find that the matrix entries will contain terms of the form $G_{f}^{3} v_{F}^{3}, G_{f}^{3} v_{F}^{4}$ and 
depending on representation, $G_{f}^{3} v_{F}^{5}$. This may help us gain further insight into fermion masses as well as high-order Lagrangian vacuum terms $\phi^{3}, \phi^{4}, \phi^{5}$.

All of this mystery cracking is the result of the detective work embarked upon at the start of Section 6 , of pulling on the tiny thread of the complex factor $i^{0.5}=\frac{1}{\sqrt{2}}(1+i)$ which arises from taking the fourth root of the minus (-) sign that emanates from the oppositely-signed electric charges of the up and down quarks, in order to form the Koide matrices (5.6) and (5.7).

\section{Vacuum-Amplified and Constituent Quark Masses}

In (5.12) through (5.14) we defined three very helpful mass values all between $604 \mathrm{MeV}$ and $635 \mathrm{MeV}$. It is natural therefore to inquire whether these "vacuum-amplified" quark masses might be related to the so-called "constituent" quark masses which specify how much mass each quark contributes to total mass of a nucleon or baryon, as opposed to the bare "current" quark masses. Specifically, recalling that these were the ingredients in the neutron plus proton mass sum, we note $M_{u} / 2$ $=302.0875673 \mathrm{MeV}, M_{d} / 2=317.2892232 \mathrm{MeV}$ in (5.12) and (5.13), which is about $1 / 3$ of the neutron and proton masses. This suggests that (5.12) to (5.14) may be related to the constituent masses of the up and down quarks which specify how much of the observed neutron and proton masses arise from each of the quarks and their interactions with the vacuum. The question we now ask, referring to the neutron and proton mass formulas (6.31), is how much does each up quark contribute, and how much does each down quark contribute, to these total masses? In other words, what are the "constituent" masses of the up quarks and down quarks in each of the neutron and proton, as opposed to their bare "current" masses?

Referring to the neutron and proton masses (6.31), for the square root terms $\sqrt{M_{u} M_{d}}$ and $\sqrt{m_{\mu} m_{d}}$, we cannot directly segregate the up quark mass contribution from that of the down quark. In these square root terms, the up and down are coequal mass contributors. So we shall allocate instead. For the term $3 \cdot \sqrt{M_{u} M_{d}}$ in the neutron mass, we allocate a $1 \cdot \sqrt{M_{u} M_{d}}$ contribution to the one up quark and a total $2 \cdot \sqrt{M_{u} M_{d}}$ contribution to the two down quarks. For the proton, we allocate $1 \cdot \sqrt{M_{u} M_{d}}$ to the one down quark and $2 \cdot \sqrt{M_{u} M_{d}}$ to the two up quarks. We similarly allocate the $\sqrt{m_{\mu} m_{d}}$ terms. But as to terms which contain $m_{u}$ alone, or $m_{d}$ alone, we segregate these and apply them directly to the up and down quark constituent masses, respectively. Thus, we identically rewrite each of (6.31) while defining respective constituent quark mass sums $U_{N}+2 D_{N}$ and $2 U_{P}+D_{P}$, as:

$$
\begin{gathered}
M_{N}=\frac{1}{2}\left(\begin{array}{c}
\sqrt{M_{u} M_{d}}+3 m_{u} \cos \theta_{1}+m_{u}-\frac{2 \sqrt{m_{\mu} m_{d}}}{3(2 \pi)^{\frac{3}{2}}}+\frac{3 m_{u}}{(2 \pi)^{\frac{3}{2}}} \\
2 \sqrt{M_{u} M_{d}}+3 m_{d} \cos \theta_{1}-\frac{4 \sqrt{m_{\mu} m_{d}}}{3(2 \pi)^{\frac{3}{2}}}-\frac{3 m_{d}}{(2 \pi)^{\frac{3}{2}}}
\end{array}\right) \equiv U_{N}+2 D_{N}, \\
M_{P}=\frac{1}{2}\left(\begin{array}{c}
2 \sqrt{M_{u} M_{d}}+3 m_{u} \cos \theta_{1}-m_{u}+\frac{4 \sqrt{m_{\mu} m_{d}}}{3(2 \pi)^{\frac{3}{2}}}-\frac{3 m_{u}}{(2 \pi)^{\frac{3}{2}}} \\
\sqrt{M_{u} M_{d}}+3 m_{d} \cos \theta_{1}+\frac{2 \sqrt{m_{\mu} m_{d}}}{3(2 \pi)^{\frac{3}{2}}}+\frac{3 m_{d}}{(2 \pi)^{\frac{3}{2}}}
\end{array}\right) \equiv 2 U_{P}+D_{P},
\end{gathered}
$$

with the up and down quark contributions respectively specified in the upper and lower lines of each of (8.1) and (8.2). That is, the above represent a deconstruction of the neutron and proton masses into the separate contribu- tions emanating from up and down quarks. We then separate out the constituent quark masses and calculate them using $\cos \theta_{1}=0.9474541242$, as follows:

$$
U_{N}=\frac{1}{2}\left(\sqrt{M_{u} M_{d}}+3 m_{u} \cos \theta_{1}+m_{u}-\frac{2 \sqrt{m_{\mu} m_{d}}}{3(2 \pi)^{\frac{3}{2}}}+\frac{3 m_{u}}{(2 \pi)^{\frac{3}{2}}}\right)=314.0092987 \mathrm{MeV},
$$




$$
\begin{aligned}
& D_{N}=\frac{1}{2}\left(\sqrt{M_{u} M_{d}}+\frac{3}{2} m_{d} \cos \theta_{1}-\frac{2 \sqrt{m_{\mu} m_{d}}}{3(2 \pi)^{\frac{3}{2}}}-\frac{3 m_{d}}{2(2 \pi)^{\frac{3}{2}}}\right)=312.7780400 \mathrm{MeV}, \\
& U_{P} \equiv \frac{1}{2}\left(\sqrt{M_{u} M_{d}}+\frac{3}{2} m_{u} \cos \theta_{1}-m_{u}+\frac{2 \sqrt{m_{\mu} m_{d}}}{3(2 \pi)^{\frac{3}{2}}}-\frac{3 m_{u}}{2(2 \pi)^{\frac{3}{2}}}\right)=310.0274283 \mathrm{MeV} . \\
& D_{P}=\frac{1}{2}\left(\sqrt{M_{u} M_{d}}+3 m_{d} \cos \theta_{1}+\frac{2 \sqrt{m_{\mu} m_{d}}}{3(2 \pi)^{\frac{3}{2}}}+\frac{3 m_{d}}{(2 \pi)^{\frac{3}{2}}}\right)=318.2171900 \mathrm{MeV} .
\end{aligned}
$$

The first expression (8.3) for $U_{N}$ is the constituent contribution of the up quark to the mass of the neutron. The second expression (8.4) for $D_{N}$ is the constituent contribution of each of the two down quarks to the mass of the neutron. $U_{P}$ in (8.5) is the constituent contribution of each of the two up quarks to the mass of the proton. Finally, $D_{P}$ in (8.6) is the constituent contribution of the down quark to the mass of the proton. One can verify that $M_{N}=U_{N}+2 D_{N}$ and $M_{P}=2 U_{P}+D_{P}$, numerically and analytically. It is important to observe that $U_{N} \neq U_{P}$ and $D_{N} \neq D_{P}$, which is to say that the constituent contribution of each quark to the mass of a nucleon is not the same for different nucleons, but rather is dependent upon the particular nucleon in question, in this case, a proton or a neutron. So the lone up quark in the neutron makes a slightly greater contribution to the overall neutron mass than each of the two down quarks, and the lone down quark in the proton makes a slightly greater contribution to the proton mass than each of the two up quarks.

This sort of context-dependent variable behavior depending upon nuclide is to be expected based not only on what we uncovered throughout [5], but more generally based on the fact that when nucleons bind together, they release binding energy, so that different nuclides have different weights per nucleon, and indeed, different nucleons within a given nuclide should be expected to have different weights from one another based on their shell characterization. Constituent mass Equations (8.3) through (8.6) tell us along these same lines, that the constituent mass contributions from each quark will differ depending upon the particular nuclide in question, and indeed, upon the particular nucleon with which a quark is associated within that nuclide. The above, (8.3) through (8.6), make the point that this type of variable mass behavior of individual quarks already starts to appear even as between the free neutron and proton.
We also see that the "vacuum-amplified" quark masses (5.12) through (5.14), are not synonymous with constituent quark masses. These vacuum-amplified masses are ingredients which are used as part of the calculation of the constituent quark masses. While the constituent quark masses vary from one nucleon and nuclide and nucleon within a nuclide to the next, the vacuum-amplified quark masses do not vary. They are mass constants (to the same degree that current quark masses are constants, recognizing mass screening) which do not change from one nucleon or nuclide to the next, and which are used as ingredients for calculating the varying constituent quark masses, as we see in (8.3) through (8.6), as well as for calculating neutron and proton masses (6.31) and nuclear weights (6.32) through (6.36).

\section{The Lagrangian Formulation of the Neutron plus Proton Mass Sum}

Now we revert to the start of Section 5, where we noted that we can connect any Koide matrix products to a Lagrangian via (4.4) and (4.5). Now that we have obtained a theoretical expression for the neutron and proton masses, it is time to backtrack using the development in Section 4 to connect these masses to their associated Lagrangian expression. This is simply to put all of the foregoing into a more formal physics context so that this is understood as going beyond simply playing with mass numbers to make them numerically fit an equation with opaque origins. We shall develop such a Lagrangian formulation for the neutron plus proton mass sum (6.6), recognizing that a Lagrangian connection for the separate masses of the neutron and proton can then be developed using Yang-Mills matrix expressions such as (5.3), (5.4), (6.3) and (7.4) of [5] to also develop a Lagrangian formulation of neutron minus proton mass difference (1.4).

Using the Pauli spin matrix $T_{2}$, a unitary rotation matrix may of course be formed using: 


$$
\begin{aligned}
\exp \left(i T_{2} \theta\right) & =1+i T_{2} \theta+\frac{1}{2 !}\left(i T_{2} \theta\right)^{2}+\frac{1}{3 !}\left(i T_{2} \theta\right)^{3}+\frac{1}{4 !}\left(i T_{2} \theta\right)^{4}+\cdots \\
& =\left(\begin{array}{ll}
1 & 0 \\
0 & 1
\end{array}\right)+\left(\begin{array}{cc}
0 & \theta \\
-\theta & 0
\end{array}\right)-\frac{1}{2 !}\left(\begin{array}{cc}
\theta^{2} & 0 \\
0 & \theta^{2}
\end{array}\right)+\frac{1}{3 !}\left(\begin{array}{cc}
0 & -\theta^{3} \\
\theta^{3} & 0
\end{array}\right)+\frac{1}{4 !}\left(\begin{array}{cc}
\theta^{4} & 0 \\
0 & \theta^{4}
\end{array}\right)+\cdots \\
& =\left(\begin{array}{cc}
1-\frac{1}{2 !} \theta^{2}+\frac{1}{4 !} \theta^{4}+\cdots & \theta-\frac{1}{3 !} \theta^{3}+\cdots \\
-\left(\theta-\frac{1}{3 !} \theta^{3}\right)+\cdots & 1-\frac{1}{2 !} \theta^{2}+\frac{1}{4 !} \theta^{4}+\cdots
\end{array}\right)=\left(\begin{array}{cc}
\cos \theta & \sin \theta \\
-\sin \theta & \cos \theta
\end{array}\right)
\end{aligned}
$$

Consequently, the square root of this rotation matrix is:

$$
\sqrt{\exp \left(i T_{2} \theta\right)}=\exp \left(\frac{1}{2} i T_{2} \theta\right)=\left(\begin{array}{cc}
\cos \frac{1}{2} \theta & \sin \frac{1}{2} \theta \\
-\sin \frac{1}{2} \theta & \cos \frac{1}{2} \theta
\end{array}\right)
$$

With this in mind we start with the expression (6.6) including the phase $\exp (i \delta)$ which we later found in (6.30) is $\exp (i \delta)=1$, and write the neutron plus proton mass sum using a square root rotation matrix as:

$$
\begin{aligned}
M_{N}+M_{P} & =\mathrm{E}_{A B} U_{1 B C} \mathrm{E}_{C A}=\mathrm{E}_{A B} \sqrt{U_{1}}{\sqrt{U_{1}}}_{C D} \mathrm{E}_{D A} \equiv \overline{\mathrm{E}}_{A B}^{\prime} \mathrm{E}_{B A}^{\prime} \\
& =3 \operatorname{Tr}\left(\begin{array}{ccc}
\sqrt[4]{M_{u} M_{d}} \exp \left(\frac{1}{2} i \delta\right) & 0 & 0 \\
0 & \sqrt{m_{u}} \cos \frac{1}{2} \theta_{1} & \sqrt{m_{u}} \sin \frac{1}{2} \theta_{1} \\
0 & -\sqrt{m_{d}} \sin \frac{1}{2} \theta_{1} & \sqrt{m_{d}} \cos \frac{1}{2} \theta
\end{array}\right) \\
& =3\left(\exp (i \delta) \sqrt{M_{u} M_{d}}+m_{u} \cos \theta_{1}+m_{d} \cos \theta_{1}\right)
\end{aligned}
$$

in combination with a rotated "electron generation matrix" $\mathrm{E}^{\prime}$ defined via left multiplication with $\sqrt{U_{1}}$ as:

$$
\begin{aligned}
& \mathrm{E}_{A B}^{\prime}=\sqrt{3}\left(\begin{array}{ccc}
\sqrt[4]{M_{u} M_{d}} \exp \left(\frac{1}{2} i \delta\right) & 0 & 0 \\
0 & \sqrt{m_{u}} \cos \frac{1}{2} \theta_{1} & \sqrt{m_{d}} \sin \frac{1}{2} \theta_{1} \\
0 & -\sqrt{m_{u}} \sin \frac{1}{2} \theta_{1} & \sqrt{m_{d}} \cos \frac{1}{2} \theta_{1}
\end{array}\right)=\sqrt{U_{1}}{ }_{A C} \mathrm{E}_{C B} \\
& =\sqrt{3}\left(\begin{array}{ccc}
\exp \left(\frac{1}{2} i \delta\right) & 0 & 0 \\
0 & \cos \frac{1}{2} \theta_{1} & \sin \frac{1}{2} \theta_{1} \\
0 & -\sin \frac{1}{2} \theta_{1} & \cos \frac{1}{2} \theta_{1}
\end{array}\right)\left(\begin{array}{ccc}
\sqrt[4]{M_{u} M_{d}} & 0 & 0 \\
0 & \sqrt{m_{u}} & 0 \\
0 & 0 & \sqrt{m_{d}}
\end{array}\right)
\end{aligned}
$$

and an adjoint matrix defined via right-multiplication with $\sqrt{U_{1}}$ as: 


$$
\begin{aligned}
& \overline{\mathrm{E}}_{A B}^{\prime} \equiv \sqrt{3}\left(\begin{array}{ccc}
\sqrt[4]{M_{u} M_{d}} \exp \left(\frac{1}{2} i \delta\right) & 0 & 0 \\
0 & \sqrt{m_{u}} \cos \frac{1}{2} \theta_{1} & \sqrt{m_{u}} \sin \frac{1}{2} \theta_{1} \\
0 & -\sqrt{m_{d}} \sin \frac{1}{2} \theta_{1} & \sqrt{m_{d}} \cos \frac{1}{2} \theta
\end{array}\right)=\mathrm{E}_{A C} \sqrt{U_{1}}{ }_{C B} \\
& =\left(\begin{array}{ccc}
\sqrt[4]{M_{u} M_{d}} & 0 & 0 \\
0 & \sqrt{m_{u}} & 0 \\
0 & 0 & \sqrt{m_{d}}
\end{array}\right)\left(\begin{array}{ccc}
\exp \left(\frac{1}{2} i \delta\right) & 0 & 0 \\
0 & \cos \frac{1}{2} \theta_{1} & \sin \frac{1}{2} \theta_{1} \\
0 & -\sin \frac{1}{2} \theta_{1} & \cos \frac{1}{2} \theta_{1}
\end{array}\right)
\end{aligned}
$$

In the above, $\cos \theta_{1}=0.9474541242$ is the empirical number found in (6.28), and $\delta=0$ is identically true as found in (6.30). The above, $\mathrm{E}_{A B}^{\prime}$ and $\overline{\mathrm{E}}_{A B}^{\prime}$, are just the Koide triplet matrix $\mathrm{E}_{A B}$ for the electron generation rotated into primed state by multiplying from the left and from the right via $\sqrt{U_{1}} \mathrm{E}_{C B}$ and $\mathrm{E}_{A C} \sqrt{U_{1}}$.

But we know from (4.4) and (4.5) that as soon as we have a Koide matrix, we can backtrack into a Lagrangian formulation. In this case, in (2.1) for a generalized Koide matrix $K_{A B}$, we are setting $m_{1}=\sqrt{M_{u} M_{d}}, m_{2}=m_{u}$ and $m_{3}=m_{d}$, and the only new feature is that we are then rotating this matrix both from the left and the right via $K^{\prime}=\sqrt{U} K$ and $\bar{K}=K \sqrt{U}$. Consequently, we may use (9.4) and (9.5) to write the mass sum $M_{N}+M_{P}$ in (9.3) in a Lagrangian formulation, using these rotated Koide matrices, via (4.4) and (4.5) as:

$$
\begin{aligned}
& M_{N}+M_{P}=-(2 \pi)^{\frac{3}{2}} \iiint \mathcal{L} \mathrm{d}^{3} x=\frac{1}{2}(2 \pi)^{\frac{3}{2}} \operatorname{Tr} \iiint \overline{\mathscr{E}}_{\mu \nu}^{\prime} \mathcal{E}^{\prime \mu \nu} \mathrm{d}^{3} x=\frac{1}{2}(2 \pi)^{\frac{3}{2}} \operatorname{Tr} \iiint \overline{\mathscr{E}}_{A B}^{\prime} \cdot \mathscr{E}_{B D}^{\prime} \mathrm{d}^{3} x \\
& =\frac{1}{2}(2 \pi)^{\frac{3}{2}} \iiint \overline{\mathcal{E}}_{A B}^{\prime} \cdot \widetilde{E}_{B A}^{\prime} \mathrm{d}^{3} x=\overline{\mathrm{E}}_{A B}^{\prime} \mathrm{E}_{B A}^{\prime}=3 \operatorname{Tr}\left(\begin{array}{ccc}
\sqrt[4]{M_{u} M_{d}} \exp \left(\frac{1}{2} i \delta\right) & 0 & 0 \\
0 & \sqrt{m_{u}} \cos \frac{1}{2} \theta_{1} & \sqrt{m_{u}} \sin \frac{1}{2} \theta_{1} \\
0 & -\sqrt{m_{d}} \sin \frac{1}{2} \theta_{1} & \sqrt{m_{d}} \cos \frac{1}{2} \theta
\end{array}\right)^{2} \\
& =3\left(\exp (i \delta) \sqrt{M_{u} M_{d}}+m_{u} \cos \theta_{1}+m_{d} \cos \theta_{1}\right)=M_{N}+M_{P}
\end{aligned}
$$

by introducing new field strength tensors defined in the manner of (4.2) as:

$$
\begin{aligned}
& \operatorname{Tr} \mathcal{G}^{\prime \mu v} \equiv-i\left(\frac{\bar{\Psi}_{u d}^{\prime}\left[\gamma^{\mu}, \gamma^{v}\right] \Psi_{u d}^{\prime}}{\sqrt{M_{u} M_{d}}}+\frac{\bar{\Psi}_{u}^{\prime}\left[\gamma^{\mu}, \gamma^{v}\right] \psi_{u}^{\prime}}{m_{u}^{\prime}}+\frac{\psi_{d}^{\prime}\left[\gamma^{\mu}, \gamma^{v}\right] \psi_{d}^{\prime}}{m_{d}^{\prime}}\right), \\
& \operatorname{Tr} \overline{\mathcal{G}}^{\prime \mu \nu} \equiv-i\left(\frac{\bar{\Psi}_{u d}^{\prime}\left[\gamma^{\mu}, \gamma^{v}\right] \Psi_{u d}^{\prime}}{{\sqrt{M_{u} M_{d}}}^{\prime}}+\frac{\bar{\Psi}_{u}^{\prime}\left[\gamma^{\mu}, \gamma^{v}\right] \psi_{u}^{\prime}}{m_{u}^{\prime}}+\frac{\psi_{d}^{\prime}\left[\gamma^{\mu}, \gamma^{v}\right] \psi_{d}^{\prime}}{m_{d}^{\prime}}\right),
\end{aligned}
$$

where the "vacuum-amplified" masses $M_{u}$ and $M_{d}$ as well as the square root mass $\sqrt{M_{u} M_{d}}$ are defined as in (5.12) to (5.14), and where the Koide mass matrices are formed for $\mathcal{E}^{\prime \mu \nu}$ using left-multiplication (9.4) and for $\overline{\mathcal{G}}^{\prime \mu \nu}$ using right-multiplication (9.5).
Referring back to Sections 2 and 4, this means that here we have set $\psi_{1}^{\prime}=\Psi_{u d}^{\prime}, \psi_{2}^{\prime}=\psi_{u}^{\prime}, \psi_{3}^{\prime}=\psi_{d}^{\prime}$ in the field strength tensor (4.2) and as just noted, $m_{1}=\sqrt{M_{u} M_{d}}$, $m_{2}=m_{u}, m_{3}=m_{d}$ in the Koide matrix (2.1), then followed the remaining development of Section 4 with the 
only addition being that we now are also employing the rotations (9.4) and (9.5) on these Koide triplet matrices. We also now have the knowledge which can be exploited for further future development, that (9.3) for the neutron plus proton mass sum specifies a special case of the very general master mass and mixing matrix $\Theta$ as specified in (6.17), see (6.20). So this gives us a hook into a Lagrangian formulation for other generations of fermion, and therefore, for formulating other charmed, strange, top and bottom-containing baryons.

As a consequence of the foregoing, the unrotated fermion eigenstates used to form (9.7) and (9.8) are a triplet $\left(\Psi_{u d}, \psi_{u}, \psi_{d}\right)$ consisting of a wavefunction for a vacuum-enhanced fermion $\Psi_{u d}$ (using upper case Greek), together with the ordinary fermion wavefunctions $\psi_{u}, \psi_{d}$ for the up and down current quarks (lower case Greek). It is the $\Psi_{u d}$ wavefunction that is responsible for generating the vast preponderance of the constituent mass contributions to the neutron plus proton mass sum, see Section 8, while $\psi_{u}, \psi_{d}$ are responsible for the current mass contributions.

Lastly, as in (4.12) through (4.14), at the nuts and bolts level, we apply the Gaussian ansatz (4.12), in the form:

$$
\begin{aligned}
& \psi_{u}(r)=u\left(\pi \lambda_{u}^{2}\right)^{-\frac{3}{4}} \exp \left(-\frac{1}{2} \frac{\left(r-r_{0}\right)^{2}}{\hbar_{u}^{2}}\right), \\
& \psi_{d}(r)=d\left(\pi \lambda_{d}^{2}\right)^{-\frac{3}{4}} \exp \left(-\frac{1}{2} \frac{\left(r-r_{0}\right)^{2}}{\pi_{d}^{2}}\right), \\
& \Psi_{u d}(r)=V\left(\pi X_{u d}^{2}\right)^{-\frac{3}{4}} \exp \left(-\frac{1}{2} \frac{\left(r-r_{0}\right)^{2}}{X_{u d}^{2}}\right),
\end{aligned}
$$

and for the reduced Compton wavelengths, converting to $\hbar=c=1$ units, we specify:

$$
\begin{aligned}
& \lambda_{u}=\hbar / m_{u} c=1 / m_{u}, \\
& \hbar_{d}=\hbar / m_{d} c=1 / m_{d}, \\
& \mathscr{X}_{u d}=\hbar / \sqrt{M_{u} M_{d}} c=1 / \sqrt{M_{u} M_{d}} .
\end{aligned}
$$

So, referring back to the discussion at the end of Section 4, as was the case with the short range of the nuclear interaction, we can indeed use the Gaussian ansatz to model fermion wavefunctions as Gaussians and obtain the fully-dressed neutron and proton masses. But to do so, in the above we are using the undressed "current" quarks $\psi_{u}, \psi_{d}$ which yielded binding energies in [1,5], together in the same Koide triplet with a vacuum-amplified quark wavefunction $\Psi_{u d}$ and associated masses and wavelengths. So here too, it is not a question of whether we can use a Gaussian ansatz, but rather, it is a question of which wavefunctions with which masses and wavelengths we need to use in the Gaussian ansatz, in order to obtain a precise concurrence with empirical data.

So, insofar as fully covered protons and neutrons are concerned, it looks as if the vacuum-amplified quarks in combination with the current quarks, are behaving as free fermions, as specified in detail in all of the foregoing. This underscores the role of the Gaussian ansatz as a modeling tool used to derive effective concurrence with empirical data, rather than as a part of the theory per se. The theory is centered on baryons being Yang-Mills magnetic monopoles, and nucleons releasing or retaining binding energies based on their resonant properties which in turn depend upon the current quark content of those nucleons. For calculations which involve the components and emissions of protons and neutrons such as their current quarks and their binding energies, the current quarks can be modeled as free fermions to obtain empirically-accurate results. For other calculations which involve the bulk behavior of protons and neutrons, accurate results may be obtained by modeling vacuum-enhanced quarks in combination with current quarks as free fermions, in the manner outlined above.

The whole point of the discussion in this Section has been to make clear that the neutron plus proton mass sum (and thus the individual neutron and proton masses) developed in this paper is not just the result of developing formulas which fit the empirical data but have unclear, opaque origins in the way that the Koide relations have also had unclear origins. Rather, as shown in (9.6) this mass sum can be formulated as the energy

$$
\begin{aligned}
M_{N}+M_{P} & =-(2 \pi)^{\frac{3}{2}} \iiint \mathfrak{2} \mathrm{d}^{3} x \\
& =\frac{1}{2}(2 \pi)^{\frac{3}{2}} \operatorname{Tr} \iiint \overline{\mathcal{E}}_{\mu v}^{\prime} \mathcal{E}^{\prime \mu v} \mathrm{~d}^{3} x
\end{aligned}
$$

arising from integrating a Lagrangian density

$\mathfrak{L}=-\frac{1}{2}{\overline{\sigma_{\mu \nu}^{\prime}}}_{\mu \nu} \mathcal{E}^{\prime \mu \nu}$ over the entirety of a three-space volume element $\mathrm{d}^{3} x$. This puts the neutron and proton masses (and by implication via $\Theta$ as specified in (6.17), other baryon masses as well) into the context of fundamental, Lagrangian-based physics, and shows how these mass formulas (as well as those of Koide) are not just coincidental numeric coincidences of unexplained origin, but truly are real physics relationships with a Lagrangian foundation.

\section{Conclusion}

In conclusion, we have shown how the Koide relationships and associated triplet mass matrices can be generalized to derive the observed sum of the free neutron and proton rest masses in terms of the up and down current quark masses and the Fermi vev to six parts in 10,000, see (5.18). This sum can then be solved for the separate 
neutron and proton masses using the neutron minus proton mass difference (1.4) earlier derived in [5], as shown in (6.22). The oppositely-signed charges of the up and down quarks are responsible for the appearance of a complex phase $\exp (\mathrm{i} \delta)$ and real rotation angle $\theta$ which leads on an independent basis to mass and mixing matrices similar to that of Cabibbo, Kobayashi and Maskawa (CKM), see (6.5) and (6.14). These can then be used to specify the neutron and proton mass relationships to unlimited accuracy as shown in (6.31) using $\theta$ as a nucleon fitting angle deduced in (6.28) from empirical data. This fitting angle is then shown in (7.5) to be related to an invariant of the CKM mixing angles within experimental errors. Also of interest is a master mass and mixing matrix developed in (6.17) which may help to interconnect all baryon and quark masses and mixing angles. The Koide generalizations developed here enable these neutron and proton mass relationships to be given a Lagrangian formulation based on neutron and proton field strength tensors that contain vacuum-amplified and current quark wavefunctions and masses, as shown in Sections 8 and 9 . In the course of development, we also uncover new Koide relationships (3.14), (3.16) and (3.17) for the neutrinos, the up quarks, and the down quarks.

\section{REFERENCES}

[1] J. R. Yablon, "Why Baryons Are Yang-Mills Magnetic Monopoles," Hadronic Journal, Vol. 35, No. 4, 2012, pp. 399-467.

http://www.hadronicpress.com/issues/HJ/VOL35/HJ-35-4 .pdf

[2] G. t'Hooft, "Magnetic Monopoles in Unified Gauge Theories," Nuclear Physics B, Vol. 79, No. 2, 1974, pp. 276-284. doi:10.1016/0550-3213(74)90486-6

[3] H. C. Ohanian, "What Is Spin?" American Journal of
Physics, Vol. 54, No. 6, 1986, pp. 500-505. doi: $10.1119 / 1.14580$

[4] http://www.tau.ac.il/ elicomay/emc.html

[5] J. R. Yablon, "Predicting the Binding Energies of the 1s Nuclides with High Precision, Based on Baryons which Are Yang-Mills Magnetic Monopoles," Journal of Modern Physics, Vol. 4 No. 4A, 2013, pp. 70-93. doi:10.4236/jmp.2013.44A010.

[6] Y. Koide, "Fermion-Boson Two-Body Model of Quarks and Leptons and Cabibbo Mixing," Lettere al Nuovo Cimento, Vol. 34, No. 8, 1982, pp. 201-205. doi:10.1007/BF02817096

[7] http://pdg.lbl.gov/2012/tables/rpp2012-sum-leptons.pdf

[8] J. R. Yablon, "Grand Unified SU(8) Gauge Theory Based on Baryons which Are Yang-Mills Magnetic Monopoles," Journal of Modern Physics, Vol. 4 No. 4A, 2013, pp. 94-120. doi:10.4236/jmp.2013.44A011

[9] http://pdg.lbl.gov/2012/listings/rpp2012-list-neutrino-pro p.pdf

[10] http://pdg.lb.gov/2012/tables/rpp2012-sum-quarks.pdf

[11] A. Rivero, "A New Koide Tuple: Strange-Charm-Bottom," 2011. http://arxiv.org/abs/1111.7232

[12] http://cerncourier.com/cws/article/cern/29651

[13] M. Cresti, G. Pasquali, L. Peruzzo, C. Pinori and G. Sartori, "Measurement of the Antineutron Mass," Physics Letters B, Vol. 177, No. 2, 1986, pp. 206-210. doi:10.1016/0370-2693(86)91058-0

[14] http://pdg.lbl.gov/2012/reviews/rpp2012-rev-ckm-matrix. pdf

[15] J. E. Kim and M.-S. Seo, "A Simple Expression of the Jarlskog Determinant," 2012. http://arxiv.org/abs/1201.3005

[16] F. Halzen and A. D. Martin, "Quarks and Leptons: An Introductory Course in Modern Particle Physics," John Wiley \& Sons, Hoboken, 1984. 